TRANSACTIONS OF THE

AMERICAN MATHEMATICAL SOCIETY

Volume 364, Number 11, November 2012, Pages 5857-5880

S 0002-9947(2012)05669-8

Article electronically published on May 7, 2012

\title{
MEROMORPHIC EXTENSIONS \\ FROM SMALL FAMILIES OF CIRCLES AND HOLOMORPHIC EXTENSIONS FROM SPHERES
}

\author{
JOSIP GLOBEVNIK
}

\begin{abstract}
Let $\mathbb{B}$ be the open unit ball in $\mathbb{C}^{2}$ and let $a, b, c$ be three points in $\mathbb{C}^{2}$ which do not lie in a complex line, such that the complex line through $a, b$ meets $\mathbb{B}$ and such that if one of the points $a, b$ is in $\mathbb{B}$ and the other in $\mathbb{C}^{2} \backslash \overline{\mathbb{B}}$ then $\langle a \mid b\rangle \neq 1$ and such that at least one of the numbers $\langle a \mid c\rangle,\langle b \mid c\rangle$ is different from 1 . We prove that if a continuous function $f$ on $b \mathbb{B}$ extends holomorphically into $\mathbb{B}$ along each complex line which meets $\{a, b, c\}$, then $f$ extends holomorphically through $\mathbb{B}$. This generalizes the recent result of L. Baracco who proved such a result in the case when the points $a, b, c$ are contained in $\mathbb{B}$. The proof is quite different from the one of Baracco and uses the following one-variable result, which we also prove in the paper: Let $\Delta$ be the open unit disc in $\mathbb{C}$. Given $\alpha \in \Delta$ let $\mathcal{C}_{\alpha}$ be the family of all circles in $\Delta$ obtained as the images of circles centered at the origin under an automorphism of $\Delta$ that maps 0 to $\alpha$. Given $\alpha, \beta \in \Delta, \alpha \neq \beta$, and $n \in \mathbb{N}$, a continuous function $f$ on $\bar{\Delta}$ extends meromorphically from every circle $\Gamma \in \mathcal{C}_{\alpha} \cup \mathcal{C}_{\beta}$ through the disc bounded by $\Gamma$ with the only pole at the center of $\Gamma$ of degree not exceeding $n$ if and only if $f$ is of the form $f(z)=a_{0}(z)+a_{1}(z) \bar{z}+\cdots+a_{n}(z) \bar{z}^{n}(z \in \Delta)$ where the functions $a_{j}, 0 \leq j \leq n$, are holomorphic on $\Delta$.
\end{abstract}

\section{The MAIN RESUlts}

Denote by $\Delta$ the open unit disc in $\mathbb{C}$. If $a \in \mathbb{C}$ and $r>0$ write $\Delta(a, r)=\{\zeta \in$ $\mathbb{C}:|\zeta-a|<r\}$. Given $\alpha \in \Delta$ the Moebius map

$$
\zeta \mapsto M_{\alpha}(\zeta)=\frac{\alpha-\zeta}{1-\bar{\alpha} \zeta}(\zeta \in \Delta)
$$

maps the circles in $\Delta$ centered at the origin to the circles

$$
\left\{\frac{\alpha-R \zeta}{1-\bar{\alpha} R \zeta}: \zeta \in b \Delta\right\}, 0<R<1,
$$

which are called the circles with the hyperbolic center $\alpha$, and we denote this family of circles by $\mathcal{C}_{\alpha}$. In particular, $\mathcal{C}_{0}$ is the family of all circles in $\Delta$ centered at the origin.

If $\Gamma$ is a circle, we denote by $c(\Gamma)$ its center; moreover, if $\Gamma \subset \Delta$ we denote by $h(\Gamma)$ its hyperbolic center, that is, the unique point such that $\Gamma \in \mathcal{C}_{h(\Gamma)}$. In the case when $\alpha \in b \Delta$, then we denote by $\mathcal{C}_{\alpha}$ the family of all circles in $\bar{\Delta}$ which pass through $\alpha$.

Received by the editors December 30, 2010 and, in revised form, January 24, 2011.

2010 Mathematics Subject Classification. Primary 32V25.

(C)2012 American Mathematical Society Reverts to public domain 28 years from publication 
We say that a continuous function $f$ on a circle $\Gamma$ extends holomorphically (meromorphically) from $\Gamma$ if it extends holomorphically (meromorphically) through the disc bounded by $\Gamma$.

If a function $f$ on $\Delta$ has the form

$$
f(z)=a_{0}(z)+a_{1}(z) \bar{z}+\cdots+a_{n}(z) \bar{z}^{n}(z \in \Delta)
$$

where $a_{i}, 0 \leq i \leq n$, are holomorphic functions on $\Delta$, then we say that $f$ is a polyanalytic function on $\Delta$ (of order $n \leq 1$ if $a_{n} \not \equiv 0$ ). Polyanalytic functions of order zero are holomorphic functions.

If $f$ is a polyanalytic function of order $\leq n$ on $\Delta$, then for each circle $\Gamma \subset \Delta$, the function $z \mapsto(z-c(\Gamma))^{n} f(z)$ extends holomorphically from $\Gamma$; in other words, $f$ has a meromorphic extension from $\Gamma$ (to the disc bounded by $\Gamma$ ) with the only pole at the center of $\Gamma$, which is of degree $\leq n$. Indeed, if $z \in b \Delta(a, r)$, then $\bar{z}=\bar{a}+r^{2} /(z-a)$, so

$$
(z-a)^{n} f(z)=(z-a)^{n}\left[a_{0}(z)+a_{1}(z)\left[\bar{a}+\frac{r^{2}}{z-a}\right]+\cdots+a_{n}(z)\left[\bar{a}+\frac{r^{2}}{z-a}\right]^{n}\right]
$$

provides the necessary holomorphic extension through $\Delta(a, r)$.

We begin with a one-variable result.

Theorem 1.1. Let $\alpha, \beta \in \bar{\Delta}, \alpha \neq \beta$, and let $n \in \mathbb{N} \cup\{0\}$. Let $f$ be a continuous function on $\bar{\Delta}$. Assume that for every circle $\Gamma \in \mathcal{C}_{\alpha} \cup \mathcal{C}_{\beta}$ the function $z \mapsto(z-$ $c(\Gamma))^{n} f(z)$ extends holomorphically from $\Gamma$. Then the function $f$ is polyanalytic of order $\leq n$ on $\Delta$ and consequently for every circle $\Gamma \subset \bar{\Delta}$ the function $z \mapsto$ $(z-c(\Gamma))^{n} f(z)$ extends holomorphically from $\Gamma$.

In [A2, M. Agranovsky obtained, in a real analytic case, a characterization of polyanalytic functions in terms of meromorphic extendibility from various families of circles. In the special case when $f$ is real analytic, Theorem 1.1 follows from his work. In the case when $n=0$, Theorem 1.1 follows from the result of A. Tumanov when $\alpha, \beta \in b \Delta$ [T1] and reduces to the results of the author when $\alpha \in \Delta, \beta \in b \Delta$ G2 and when $\alpha, \beta \in \Delta$ G3].

Given $a \in \mathbb{C}^{2}$ we denote by $\mathcal{L}(a)$ the family of all complex lines passing through $a$. Given $b \in \mathbb{C}^{2}, b \neq a$, we denote by $\Lambda(a, b)$ the complex line passing through $a$ and $b$. We denote by $\mathbb{B}$ the open unit ball in $\mathbb{C}^{2}$. Using Theorem 1.1 we prove

Theorem 1.2. Let $a, b$ be two points in $\mathbb{C}^{2}$ such that $\Lambda(a, b)$ meets $\mathbb{B}$. Suppose that if one of the points $a, b$ is in $\mathbb{B}$ and the other in $\mathbb{C}^{2} \backslash \overline{\mathbb{B}}$, then $\langle a \mid b\rangle \neq 1$. Assume that a continuous function $f$ on $b \mathbb{B}$ extends holomorphically into $\mathbb{B}$ along every complex line $L \in \mathcal{L}(a) \cup \mathcal{L}(b)$. Then for any $c \in \Lambda(a, b) \cap \mathbb{B}$, the function $f$ extends holomorphically into $\mathbb{B}$ along any complex line $L \in \mathcal{L}(c)$.

L. Baracco B2 recently proved a conjecture of M. Agranovsky [A1]: If $a, b, c \in \mathbb{B}$ do not lie on a complex line, then $\mathcal{L}(a) \cup \mathcal{L}(b) \cup \mathcal{L}(c)$ is a test family for holomorphic extendibility for $C(b \mathbb{B})$; that is, if $f \in C(b B)$ extends holomorphically into $\mathbb{B}$ along each complex line $L \in \mathcal{L}(a) \cup \mathcal{L}(b) \cup \mathcal{L}(c)$ where the points $a, b, c$ are in $\mathbb{B}$ and do not lie on a complex line, then $f$ extends holomorphically through $\mathbb{B}$. Another attempt to prove the conjecture was presented in [A3] by M. Agranovsky who later found that the proof in $\mathrm{A} 3$ is incomplete. Our proof of Theorem 1.2 provides a new, different proof of the result of Baracco. Indeed, Theorem 1.2 implies that $f$ extends into $\mathbb{B}$ along every complex line that meets $\mathbb{B}$ and so $f$ extends holomorphically 
through $\mathbb{B}[\mathrm{AV}], \underline{\mathrm{S}}$. Since in our Theorem 1.2 the points $a, b$ do not have to lie in $\mathbb{B}$ we get a more general result:

Corollary 1.3. Let $a, b, c$ be points in $\mathbb{C}^{2}$ which do not lie on a complex line, such that $\Lambda(a, b)$ meets $\mathbb{B}$ and such that if one of the points $a, b$ lies in $\mathbb{B}$, the other in $\mathbb{C}^{2} \backslash$ $\overline{\mathbb{B}}$, then $\langle a \mid b\rangle \neq 1$ and such that at least one of the numbers $\langle a \mid c\rangle,\langle b \mid c\rangle$ is different from 1. Then $\mathcal{L}(a) \cup \mathcal{L}(b) \cup \mathcal{L}(c)$ is a test family for holomorphic extendibility for $C(b \mathbb{B})$.

Remark. For functions $f$ in $\mathcal{C}^{\infty}(b \mathbb{B}), \mathcal{L}(a) \cup \mathcal{L}(b)$ above is a test family for holomorphic extendibility, that is, the complex lines through two points suffice G3]. This is not true for functions in $\mathcal{C}^{k}(b \mathbb{B})$ G3. Corollary 1.3 implies that if $N+1$ points in the open unit ball of $\mathbb{C}^{N}$ do not lie in an $(N-1)$ dimensional complex plane, then the complex lines passing through these points form a test family for holomorphic extendibility for continuous functions on the unit sphere in $\mathbb{C}^{N}$. It is known that the complex lines through two points in the unit ball suffice for functions of class $\mathcal{C}^{\infty}$ for any dimension $N$ G3 .

\section{Poles at the hyperbolic Centers}

We begin by a simple, but important observation of M. Agranovsky [A2]

Proposition 2.1. Let $\Gamma \subset \Delta$ be a circle bounding the open disc $D$ which is not centered at 0 . The rational extension of $z \mapsto \varphi(z)=1-|z|^{2}$ from $\Gamma$ has one zero in $D$, a single zero at $h(\Gamma)$, and one pole in $D$, a simple pole at $c(\Gamma)$.

Proof. If $\Gamma=b \Delta(a, r)$, then $z \in \Gamma$ implies that $\bar{z}=\bar{a}+r^{2} /(z-a)$, so

$$
z \mapsto 1-|z|^{2}=1-z\left[\bar{a}+\frac{r^{2}}{z-a}\right]
$$

is a rational function which has one pole $a$ in $D$, which proves the second statement. To prove the first statement, write

$$
\Gamma=\left\{\frac{\alpha-R \zeta}{1-\bar{\alpha} R \zeta}: \zeta \in b \Delta\right\},
$$

where $\alpha \in \Delta, \alpha \neq 0$, and $0<R<1$. If $\zeta \in b \Delta$, then

$$
1-\left|\frac{\alpha-R \zeta}{1-\bar{\alpha} R \zeta}\right|^{2}=\frac{\left(1-R^{2}\right)\left(1-|\alpha|^{2}\right) \zeta}{(1-\bar{\alpha} R \zeta)(\zeta-\alpha R)}
$$

which has one zero at $\zeta=0$ and one zero at $\zeta=\infty$, which implies that the rational extension of $\varphi$ from $\Gamma$ has one zero at $z=\alpha$ and the other zero at $z=1 / \bar{\alpha}$. which proves the first statement,

Remark. If $\Gamma \subset \bar{\Delta}$ meets $b \Delta$, that is, if $\Gamma \in \mathcal{C}_{\alpha}$ with $\alpha \in b \Delta$, then the rational extension of $\varphi$ from $\Gamma$ has one pole in $D$, a single pole at $c(\Gamma)$, no zero in $D$ and a double zero at $\alpha$. To see this, assume with no loss of generality that $\alpha=1$ and let 


$$
\begin{aligned}
\Gamma=\{(1-r)+r \zeta: \zeta \in b \Delta\}, & \text { where } 0<r<1 . \text { Then, for } \zeta \in b \Delta, \\
1-|(1-r)+r \zeta|^{2} & =1-(1-r)^{2}-r(1-r) \zeta-r(1-r) / \zeta-r^{2} \\
& =\frac{\zeta^{2}\left(r^{2}-r\right)-2 \zeta\left(r^{2}-r\right)+r^{2}-r}{\zeta} \\
& =\frac{r(r-1)(\zeta-1)^{2}}{\zeta},
\end{aligned}
$$

which has a double zero at $\zeta=1$. This, in particular, implies that if $\Gamma \in \mathcal{C}_{\alpha}$, the function $\zeta \mapsto(z-\alpha)^{2} /\left(1-|z|^{2}\right)$, defined an $\Gamma \backslash\{\alpha\}$, extends continuously to $\Gamma$.

Given a continuous function $f$ on $\bar{\Delta}$ and $n \in \mathbb{N}$, set

$$
F(z)=\frac{f(z)}{\left(1-|z|^{2}\right)^{n}} \text {. }
$$

Given $\gamma \in \bar{\Delta}$ consider the following two conditions $(\mathrm{H})$ and $(\mathrm{C})$ :

if $\gamma \in \Delta$, then $(z-\gamma)^{n} F(z)$ extends holomorphically from each $\Gamma \in \mathcal{C}_{\gamma}$,

if $\gamma \in b \Delta$, then $(z-\gamma)^{2 n} F(z)$ extends holomorphically from each $\left.\Gamma \in \mathcal{C}_{\gamma},\right\}$

If $\alpha \in \Delta$ and $\Gamma \in \mathcal{C}_{\alpha}$, then by the preceding discussion $(z-c(\Gamma))^{n} f(z)$ extends holomorphically from $\Gamma$ if and only if $(z-\alpha)^{n} F(z)$ extends holomorphically from $\Gamma$. Moreover, if $\alpha \in b \Delta$ and $\Gamma \in \mathcal{C}_{\alpha}$, then $(z-c(\Gamma))^{n} f(z)$ extends holomorphically from $\Gamma$ if and only if $(z-\alpha)^{2 n} F(z)$ extends holomorphically from $\Gamma$. This gives

Lemma 2.2. Let $f$ be a continuous function on $\bar{\Delta}$ and let $n \in \mathbb{N}$. Let

$$
F(z)=\frac{f(z)}{\left(1-|z|^{2}\right)^{n}} \quad(z \in \Delta) .
$$

Then for each $\gamma \in \bar{\Delta},(H)$ and $(C)$ are equivalent.

Note that if $\alpha \in \Delta$, then $(z-\alpha)^{n} F(z)$ extends holomorphically from each $\Gamma \in \mathcal{C}_{\alpha}$ if and only if $z^{n}\left(F \circ M_{\alpha}\right)(z)$ extends holomorphically from each $\Gamma \in \mathcal{C}_{0}$.

Assume for a moment that we have proved Theorem 1.1 in the following cases:

(i) $\alpha \in \Delta, \alpha \neq 0, \beta=0$,

(ii) $\alpha \in b \Delta, \beta=0$,

(iii) $\alpha=-1, \beta=1$,

that is, that we have proved that if $(\mathrm{C})$ holds for $\gamma=\alpha, \gamma=\beta$ with $\alpha, \beta$ as in (i), (ii), (iii), then (C) holds for every $\gamma \in \bar{\Delta}$, or, equivalently, if (H) holds for $\gamma=\alpha, \gamma=\beta$ with $\alpha, \beta$ as in (i), (ii), (iii), then (H) holds for every $\gamma \in \bar{\Delta}$.

Assume now that $\alpha \in \Delta, \beta \in \Delta, \beta \neq \alpha$, and that (C) holds for $\gamma=\alpha, \gamma=\beta$. By Lemma 2.2, (H) holds for $\gamma=\alpha, \gamma=\beta$. This means that both

$$
z^{n}\left(F \circ M_{\alpha}\right)(z) \text { and } z^{n}\left(F \circ M_{\beta}\right)(z)
$$

extend holomorphically from each $\Gamma \in \mathcal{C}_{0}$. In particular,

$$
z^{n}\left(F \circ M_{\alpha}\right) \circ\left(M_{\alpha}^{-1} \circ M_{\beta}\right)(z)
$$

extends holomorphically from each $\Gamma \in \mathcal{C}_{0}$, so for some $\delta \neq 0, z^{n}\left(F \circ M_{\alpha}\right)\left(M_{\delta}(z)\right)$ extends holomorphically from every $\Gamma \in \mathcal{C}_{0}$. Since the function $z \mapsto\left(1-|z|^{2}\right)^{n}(F \circ$ $\left.M_{\alpha}\right)(z)$ is also continuous on $\bar{\Delta}$, Theorem 1.1 in case (i) above implies that for each $\omega \in \Delta$ the function $z^{n}\left(F \circ M_{\omega}\right)(z)$ extends holomorphically from each $\Gamma \in \mathcal{C}_{0}$, 
which means that $(\mathrm{H})$ holds for every $\gamma$ and consequently $(\mathrm{C})$ holds for every $\gamma$. This shows that Theorem 1.1 holds for every $\alpha \in \Delta$ and every $\beta \in \Delta, \beta \neq \alpha$.

Assume now that (C) holds for $\gamma=\alpha, \gamma=\beta$, where $\alpha \in \Delta, \beta \in b \Delta$. By Lemma 2.2, $(\mathrm{H})$ holds for $\gamma=\alpha, \gamma=\beta$. In particular, $z^{n}\left(F \circ M_{\alpha}\right)(z)$ extends holomorphically for each $\Gamma \in \mathcal{C}_{0}$ and $(z-\beta)^{2 n} F(z)$ extends holomorphically from each $\Gamma \in \mathcal{C}_{\beta}$. It follows that $(z-\beta)^{2 n}\left(F \circ M_{\alpha}\right)\left(M_{\alpha}^{-1}(z)\right)$ extends holomorphically from each $\Gamma \in \mathcal{C}_{\beta}$, which means that $w \mapsto\left(M_{\alpha}(w)-M_{\alpha} M_{\alpha}^{-1} \beta\right)^{2 n}\left(F \circ M_{\alpha}\right)(w)$ extends holomorphically from each $\Gamma \in \mathcal{C}_{M_{\alpha}^{-1}(\beta)}$, which is the same as to say that $\left(w-M_{\alpha}^{-1}(\beta)^{2 n}\left(F \circ M_{\alpha}\right)(w)\right.$ extends holomorphically from each $\Gamma \in \mathcal{C}_{M_{\alpha}^{-1}(\beta)}$, that is, $F \circ M_{\alpha}$ satisfies (H) for $\gamma=0$ and for $\gamma=M_{\alpha}^{-1}(\beta)$. By Theorem 1.1 in case (ii) above it follows that $(\mathrm{H})$ holds for $F \circ M_{\alpha}$ for every $\gamma$, that is, $z^{n}\left(F \circ M_{\alpha}\right)\left(M_{\omega}(z)\right)$ extends from $\mathcal{C}_{0}$ for every $\omega$, so for every $\delta \in \Delta, \quad z^{n}\left(F \circ M_{\delta}\right)(z)$ extends holomorphically from each $\Gamma$ in $\mathcal{C}_{0}$, that is, $(\mathrm{H})$ and consequently (C) holds for every $\gamma$.

In the same way we show that if $\alpha, \beta \in b \Delta$ and if (C) holds for $\gamma=\alpha, \gamma=\beta$, then, using Theorem 1.1 in case (iii) above we see that (C) holds for every $\gamma \in \bar{\Delta}$.

All this shows that, after a rotation if necessary, it is enough to prove Theorem 1.1 in the cases when

$$
\begin{gathered}
\alpha=t, \quad 0<t<1, \quad \text { and } \beta=0, \\
\alpha=1, \quad \beta=0 \\
\alpha=-1, \quad \beta=1 .
\end{gathered}
$$

Suppose that we have done this. Then we have also proved

Theorem 2.3. Let $F$ be a continuous function on $\Delta$ such that $z \mapsto\left(1-|z|^{2}\right)^{n} F(z)$ is continuous on $\bar{\Delta}$. Let $\alpha, \beta \in \bar{\Delta}, \alpha \neq \beta$ and assume that $(H)$ holds for $\gamma=\alpha$ and $\gamma=\beta$. Then

$$
F(z)=\frac{f(z)}{\left(1-|z|^{2}\right)^{n}} \quad(z \in \Delta),
$$

where $f$ is polyanalytic of degree $\leq n$. In particular, $(H)$ holds for every $\gamma \in \bar{\Delta}$.

\section{Proof of Theorem 1.2 Assuming Theorem 1.1}

In this section we deduce Theorem 1.2 from Theorem 2.3. Along the way we describe, for a given complex line $\Lambda$ in $\mathbb{C}^{2}$ that meets $\mathbb{B}$, those continuous functions on $b \mathbb{B}$ that extend holomorphically into $\mathbb{B}$ along each complex line $L \in \mathcal{L}(c), c \in$ $\Lambda \cap \mathbb{B}$. We already know that each such function which is of class $\mathcal{C}^{\infty}$ necessarily extends holomorphically through $\mathbb{B}$.

So let $a, b$ be two points in $\mathbb{C}^{2}$ such that $\Lambda(a, b)$ meets $\mathbb{B}$, and suppose that if one of the points is in $\mathbb{B}$, the other is in $\mathbb{C}^{2} \backslash \overline{\mathbb{B}}$, then $\langle a \mid b\rangle \neq 1$. Assume that $f \in C(b \mathbb{B})$ extends holomorphically into $\mathbb{B}$ along every complex line $L \in \mathcal{L}(a) \cup \mathcal{L}(b)$.

As in G3, we use Moebius transforms to show that it is enough to prove the statement of Theorem 1.2 in the special case when $\Lambda(a, b)$ is the $z$-axis and in one of the following two cases:

(i) $a=\left(t_{1}, 0\right), b=\left(t_{2}, 0\right)$, where $0 \leq t_{1}<t_{2}<\infty, t_{2} \neq 1 / t_{1}$,

(ii) $a=(1,0), b=(-1,0)$.

As in G3] or [A1] we now use the Fourier series decomposition and averaging to reduce the problem in $\mathbb{C}^{2}$ to a series of one-variable problems. For each $z \in \Delta$, 
write the Fourier series

$$
f\left(z, e^{i \theta} \sqrt{1-|z|^{2}}\right) \sim \sum_{n=-\infty}^{\infty} c_{n}(z)\left(e^{i \theta} \sqrt{1-|z|^{2}}\right)^{n}
$$

so that

$$
f(z, w) \sim \sum_{n=-\infty}^{\infty} c_{n}(z) w^{n} \quad((z, w) \in b B, w \neq 0) .
$$

Clearly the coefficients

$$
c_{n}(z)=\left(\frac{1}{\sqrt{1-|z|^{2}}}\right)^{n} \frac{1}{2 \pi} \int_{-\pi}^{\pi} e^{-i n \theta} f\left(z, e^{i \theta} \sqrt{1-|z|^{2}}\right) d \theta
$$

are continuous on $\Delta$, and if $n<0$ they extend continuously to $\bar{\Delta}$ with zero boundary values.

If $z_{0} \in \mathbb{C}$ and if $f$ extends holomorphically into $B$ along each complex line passing through $\left(z_{0}, 0\right)$, then in the sum (3.2) the same holds for each term:

$$
\Psi_{n}(z, w)=\frac{1}{2 \pi} \int_{-\pi}^{\pi} e^{-i n \theta} f\left(z, e^{i \theta} w\right) d \theta=w^{n} c_{n}(z),
$$

a continuous function on $b \mathbb{B}$. The converse also holds: If each term $w^{n} c_{n}(z)$ in (3.2) extends holomorphically into $\mathbb{B}$ along each complex line through $\left(z_{0}, 0\right)$, then the same holds for $f$. This is so since $f$ is uniformly continuous on $b \mathbb{B}$ and so the family of functions $e^{i \theta} \mapsto f\left(z, e^{i \theta} \sqrt{1-|z|^{2}}\right), \quad z \in \bar{\Delta}$ is uniformly equicontinuous on $b \Delta$. The proof of Fejér's theorem $[\mathrm{H}]$ shows in such a case that $f\left(z, e^{i \theta} \sqrt{1-|z|^{2}}\right)$ is the limit of Cezaro means of the Fourier series (3.1) which is uniform with respect to $z \in \bar{\Delta}$.

Notice that $w^{n} c_{n}(z)$ extends holomorphically into $\mathbb{B}$ along each $\Lambda \in \mathcal{L}((t, 0))$ if and only if $(z-t)^{n} c_{n}(z)$ extends holomorphically from each $\Gamma \in \mathcal{C}_{t}$ in the case when $0 \leq t \leq 1$ and from each $\Gamma \in \mathcal{C}_{1 / t}$ in the case when $1<t<\infty$ G3].

We will now apply Theorem 2.3 to each $c_{n}$. If $n \leq 0$, then $c_{n}$ is continuous on $\bar{\Delta}$ and vanishes identically on $b \Delta$ if $n<0$. If $n \geq 0$, then we know that $\left(1-|z|^{2}\right)^{n / 2} c_{n}(z)$ extends continuously through $\bar{\Delta}$ and so the same holds for $(1-$ $\left.|z|^{2}\right)^{n} c_{n}(z)$.

Assume now that $f$ extends holomorphically into $\mathbb{B}$ along each $L \in \mathcal{L}(a) \cup \mathcal{L}(b)$, where $a, b$ are as in (i). If $n<0$ this implies that $c_{n}$ extends holomorphically from each circle belonging to $\mathcal{C}_{\tau_{1}} \cup \mathcal{C}_{\tau_{2}}$, where $0 \leq \tau_{1}<\tau_{2} \leq 1$, and since $c_{n}$ extends continuously to $\bar{\Delta}$ it follows by the main results of [G2], G3] that $c_{n}$ is holomorphic on $\Delta$. Since it is continuous on $\bar{\Delta}$ and vanishes identically on $b \Delta$ it follows that it vanishes identically on $\bar{\Delta}$.

Now, let $n>0$. Now again, we have that $\left(z-\tau_{j}\right)^{n} c_{n}(z)$ extends holomorphically from each $\Gamma \in \mathcal{C}_{\tau_{j}}, j=1,2$, which, by Theorem 2.3 implies that

$$
c_{n}(z)=\frac{g_{n}(z)}{\left(1-|z|^{2}\right)^{n}} \quad(z \in \Delta),
$$

where the function $g_{n}$ is polyanalytic of order $\leq n$ on $\Delta$ and consequently, for every $\gamma \in \Delta, \quad(z-\gamma)^{n} c_{n}(z)$ extends holomorphically from each $\Gamma \in \mathcal{C}_{\gamma}$, which implies that for each $n>0$ and for each $\gamma \in \Delta$ the function $w^{n} c_{n}(z)$ extends into $\mathbb{B}$ holomorphically along each $L \in \mathcal{L}((\gamma, 0))$ and hence, by the preceding discussion, the same holds for $f$. 
Similar reasoning applies in the case (ii). This completes the proof of Theorem 1.2 .

One should mention that the idea of multiplying $c_{n}$ with $\left(1-|z|^{2}\right)^{n}$ to achieve the regularity at the boundary and thus shifting the poles from the hyperbolic centers to the centers is due to M. Agranovsky [A1], A2].

Remark. On $b \mathbb{B}$ we have $|w|^{2}=1-|z|^{2}$, so it follows that, for $n>0$,

so

$$
c_{n}(z)=\frac{g_{n}(z)}{w^{n} \bar{w}^{n}}
$$

$$
w^{n} c_{n}(z)=\frac{g_{n}(z)}{\bar{w}^{n}}=\frac{g_{n 0}(z)+g_{n_{1}}(z) \bar{z}+\cdots+g_{n n}(z) \bar{z}^{n}}{\bar{w}^{n}} .
$$

It is easy to check directly that for each $k, 0 \leq k \leq n$, and for each complex line $L \in \mathcal{L}((\gamma, 0))$, where $\gamma \in \Delta$, different from the $z$-axis, the function

$$
(z, w) \mapsto \frac{\bar{z}^{k}}{\bar{w}^{n}}=w^{n} \frac{\bar{z}^{k}}{\left(1-|z|^{2}\right)^{n}}
$$

extends holomorphically into $B$ along $L$. This is so since

$$
z \mapsto(z-\gamma)^{n} \frac{\bar{z}^{k}}{\left(1-|z|^{2}\right)^{n}}
$$

extends holomorphically from each circle $\Gamma \in \mathcal{C}_{\gamma}$. In fact, the holomorphic extension of $\bar{z}$ from $\Gamma$ has a pole of order 1 at $c(\Gamma)$ and the same holds for $1-|z|^{2}$. On the other hand, the rational extension of $1-|z|^{2}$ from $\Gamma$ has the only zero at $\gamma$.

By the preceding reasoning we proved

Corollary 3.1. Let $f \in C(b \mathbb{B})$ and let

$$
f(z, w) \sim \sum_{-\infty}^{\infty} c_{n}(z) w^{n}
$$

be its Fourier series. The following are equivalent:

(i) $f$ extends holomorphically into $\mathbb{B}$ along each complex line that meets $\Delta \times\{0\}$.

(ii) For each $n \in Z,(z, w) \mapsto w^{n} c_{n}(z)$ extends holomorphically into $\mathbb{B}$ along each complex line that meets $\Delta \times\{0\}$.

(iii) If $n<0$, then $c_{n}(z) \equiv 0$ and if $n \geq 0$, then the function $(z, w) \mapsto c_{n}(z) w^{n}$, continuous on $b B$ has the form

$$
c_{n}(z) w^{n}=c_{n 0}(z) \frac{1}{\bar{w}^{n}}+c_{n 1}(z) \frac{\bar{z}}{\bar{w}^{n}}+c_{n n}(z) \frac{\bar{z}^{n}}{\bar{w}^{n}},
$$

where $c_{n 0}, c_{n 1}, \ldots, c_{n n}$ are holomorphic functions on $\Delta$.

Obviously, by Theorem 1.2, for either (i) or (ii) to hold it is enough that it holds for complex lines through two points of $\Delta \times\{0\}$.

Remark. When we want to construct examples we must take into account that the functions $(z, w) \mapsto w^{n} c_{n}(z)$ must be continuous on $b \mathbb{B}$. For instance, the following standard example is of this sort:

$$
\frac{w^{2}}{\bar{w}}=w^{3} \cdot \frac{1}{1-|z|^{2}}=\frac{1}{\bar{w}^{3}}\left(1-|z|^{2}\right)^{2}=\frac{1}{\bar{w}^{3}}\left(1-2 z \bar{z}+z^{2} \bar{z}^{2}\right) .
$$


Remark. We present another example, due to M. Agranovsky. Given $\alpha, \beta \in \Delta$, $\alpha \neq \beta$, the function

$$
\varphi(z)=\frac{(z-\alpha)(z-\beta)}{1-|z|^{2}} \quad(z \in \Delta)
$$

extends holomorphically from every $\Gamma \in \mathcal{C}_{\alpha} \cup \mathcal{C}_{\beta}$.

Now, let $1<t_{1}<t_{2}<\infty$. The function

$$
(z, w) \mapsto \begin{cases}w^{3} \frac{\left(z-1 / t_{1}\right)\left(z-1 / t_{2}\right)}{1-|z|^{2}}=\frac{w^{2}}{w}\left(z-1 / t_{1}\right)\left(z-1 / t_{2}\right) & (w \neq 0), \\ 0 & (w=0)\end{cases}
$$

is continuous on $b \mathbb{B}$ and extends holomorphically into $\mathbb{B}$ along any complex line meeting $\Delta \times\{0\}$ as well as along any complex line in $\mathcal{L}\left(\left(t_{1}, 0\right)\right) \cup \mathcal{L}\left(\left(t_{2}, 0\right)\right)$, yet does not extend holomorphically through $\mathbb{B}$. Obviously, finitely many points on the $z$-axis outside $\overline{\mathbb{B}}$ are also possible.

\section{Towards the Beginning of the Proof of Theorem 1.1}

We have already seen that it is enough to prove Theorem 1.1 in cases (2.2), (2.3) and (2.4). We first look at (2.2). In this case we have to prove

Theorem 4.1. Suppose that $0<t<1$ and let $n \in \mathbb{N} \cup\{0\}$. Suppose that $f$ is a continuous function on $\bar{\Delta}$ such that for each circle $\Gamma \in \mathcal{C}_{0} \cup \mathcal{C}_{t}$, the function $f \mid \Gamma$ extends meromorphically through the disc bounded by $\Gamma$ with the only pole of order $\leq n$ at the center of $\Gamma$. Then $f$ is of the form

$$
f(z)=a_{0}(z)+a_{1}(z) \bar{z}+\cdots+a_{m}(z) \bar{z}^{m},
$$

where $m \leq n$ and where $a_{j}, 0 \leq j \leq m$, are holomorphic functions on $\Delta$.

The proof of Theorem 4.1 will be our major task. We will provide a detailed proof. At the end we will indicate how to modify the proof to treat (2.3) and (2.4).

As in G2, G3] we shall use semiquadrics introduced in [AG] and G1] to transform the problem into a problem about holomorphic extensions of CR functions on surfaces consisting of these semiquadrics. The principal idea to apply the reasoning of $\mathrm{H}$. Lewy and H. Rossi in this context is due to A. Tumanov and is described in T1, and, for holomorphic extensions, is applied in G2, G3. There, the final step was to apply the Liouville theorem to conclude that the main function is constant in the second variable. The proof is considerably more complicated in the case of meromorphic extensions, where we apply the generalized Liouville theorem to conclude that the function is a polynomial in the second variable. In G2, G3] we were dealing with holomorphic extensions to semiquadrics where the maximum principle assured continuous dependence of extensions on the parameter. Now we will be dealing with meromorphic extensions where we do not have the maximum principle, so we will need the following preliminary.

Lemma 4.1. Let $I \subset \mathbb{R}$ be an interval. Let $n \in \mathbb{N}$ and let $(\zeta, t) \mapsto \Phi(\zeta, t)$ be a continuous function on $b \Delta \times I$ such that for each $t \in I$ the function $\zeta \mapsto \Phi(\zeta, t)$ extends holomorphically through $\Delta \backslash\{0\}$ and has a pole at 0 of degree $\leq n$. Denote the extension by $\tilde{\Phi}$. Then

$$
\tilde{\Phi}(\zeta, t)=\frac{d_{0}(t)}{\zeta^{n}}+\cdots+\frac{d_{n-1}(t)}{\zeta}+\Theta(\zeta, t) \quad(\zeta \in \bar{\Delta} \backslash\{0\}),
$$


where the functions $d_{j}$ are continuous on $I, \Theta$ is continuous on $\bar{\Delta} \times I$, and for each $t \in I, \quad \zeta \mapsto \Theta(\zeta, t)$ is holomorphic on $\Delta$.

Proof. The function $(\zeta, t) \mapsto \Psi(\zeta, t)=\zeta^{n} \Phi(\zeta, t)$ is continuous on $b \Delta \times I$ and for each $t, \zeta \mapsto \Psi(\zeta, t)$ extends holomorphically through $\Delta$. Denote this extension by $\tilde{\Psi}$. We have

$$
\tilde{\Psi}(\zeta, t)=d_{0}(t)+d_{1}(t) \zeta+\cdots+d_{n-1}(t) \zeta^{n-1}+\zeta^{n} \Theta(\zeta, t) \quad(\zeta \in \bar{\Delta}),
$$

where for each $j, 0 \leq j \leq n-1$,

$$
d_{j}(t)=\frac{1}{2 \pi} \int_{-\pi}^{\pi} e^{-i j \theta} \Psi\left(e^{i \theta}, t\right) d \theta
$$

is continuous on $I$ and where for each $t \in I$, the function $\zeta \mapsto \Theta(\zeta, t)$ is holomorphic on $\Delta$. The function

$$
(\zeta, t) \mapsto \Theta(\zeta, t)=\frac{\Psi(\zeta, t)}{\zeta^{n}}-\frac{d_{0}(t)}{\zeta^{n-1}}-\cdots-\frac{d_{n-1}(t)}{\zeta} \quad(\zeta \in b \Delta)
$$

is continuous on $b \Delta \times I$ and so by the maximum principle it follows that it is continuous on $\bar{\Delta} \times I$. This completes the proof.

\section{The GeOMetry of SEMiquadrics, Part 1}

Given $a \in \mathbb{C}$ and $r>0$ let

$$
\begin{aligned}
\Lambda(a, r) & =\left\{(z, w) \in \mathbb{C}^{2}:(z-a)(w-\bar{a})=r^{2}, 0<|z-a|<r\right\} \\
& =\left\{\left(z, \bar{a}+\frac{r^{2}}{z-a}\right): 0<|z-a|<r\right\}
\end{aligned}
$$

be the semiquadric associated with the circle $b \Delta(a, r)$. Write $\Sigma=\{(\zeta, \bar{\zeta}): \zeta \in \mathbb{C}\}$. $\Lambda(a, r)$ is a closed complex submanifold of $\mathbb{C}^{2} \backslash \Sigma$ which is attached to $\Sigma$ along $b \Lambda(a, r)=\{(\zeta, \bar{\zeta}): \quad \zeta \in b \Delta(a, r)\}$. The crucial property of these semiquadrics which will connect our problem with a problem in CR geometry is the following: $A$ continuous function $g$ extends meromorphically from a circle $b \Delta(a, r)$ with the pole of degree $\leq n$ at $a$ if and only if the function $G$, defined on $b \Lambda(a, r)$ by $G(\zeta, \bar{\zeta})=$ $g(\zeta)(\zeta \in b \Delta(a, r))$, extends holomorphically through $\Lambda(a, r)$ and has a pole of degree $\leq n$ at the point at infinity. In fact, if we denote by the letter $\tilde{g}$ the holomorphic extension of $g$ through $\Delta(a, r) \backslash\{a\}$ the function

$$
\tilde{G}\left(z, \bar{a}+\frac{r^{2}}{z-a}\right)=\tilde{g}(z) \quad(z \in \bar{\Delta}(a, r) \backslash\{a\})
$$

provides the necessary holomorphic extension of $G$ through $\Lambda(a, r)$. In our case, we have two families of circles:

$$
\mathcal{C}_{0}=\{b \Delta(0, R): 0<R \leq 1\}
$$

and

$$
\mathcal{C}_{t}=\{b \Delta(T, \rho(T)): 0 \leq T \leq 1\},
$$

where

$$
\rho(T)=\sqrt{(T-t)(T-1 / t)}
$$

G3], and consequently we will deal with two families of semiquadrics: the first family $\{\Lambda(0, R), 0<R \leq 1\}$ and the second family $\{\Lambda(T, \sqrt{(T-t)(T-1 / t)}): 0 \leq$ $T<t\}$. Our function $F(\zeta, \bar{\zeta})=f(\zeta)(\zeta \in \bar{\Delta})$ extends holomorphically to each 
semiquadric of each family and these extensions will then define a CR function on a hypersurface in $\mathbb{C}^{2}$ that we study and describe in detail.

In the first family the semiquadrics are pairwise disjoint. Let $L$ be the union of their closures in $\mathbb{C} \times \mathbb{C}$ :

$$
L=\bigcup_{0<R \leq 1}[\Lambda(0, R) \cup b \Lambda(0, R)]
$$

In $[\mathbb{C} \backslash\{0\}] \times \mathbb{C}$, the set $L$ is a smooth hypersurface with piecewise smooth boundary consisting of $\Lambda(0,1)$ and $\{(\zeta, \bar{\zeta}): \zeta \in \bar{\Delta} \backslash\{0\}\}$.

In the second family all semiquadrics contain the point $(t, 1 / t)$ but otherwise are pairwise disjoint, that is, the sets $\Lambda(T, \rho(T)) \backslash\{(t, 1 / t)\}$ are pairwise disjoint. The closure of their union in $[\mathbb{C} \backslash\{t\}] \times \mathbb{C}$, that is, the set

$$
N=\bigcup_{0 \leq T<t}[\Lambda(T, \rho(T)) \cup b \Lambda(T, \rho(T))] \backslash\{(t, 1 / t)\}
$$

is a smooth surface in $[\mathbb{C} \backslash\{t\}] \times \mathbb{C}$ with piecewise smooth boundary consisting of $\Lambda(0,1)$ and $\{(\zeta, \bar{\zeta}): \zeta \in \bar{\Delta} \backslash\{t\}\}$.

\section{The geometry of Semiquadrics, Part 2}

We now fix $z \in \Delta \backslash\{0\}$ and describe how the semiquadrics $\Lambda(0, R), 0<R \leq 1$, and $\Lambda(T, \rho(T)), 0 \leq T<t$, intersect the complex line $\{z\} \times \mathbb{C}$. Each semiquadric intersects $\{z\} \times \mathbb{C}$ at at most one point. For a semiquadric from the first family the intersection $(z, v(R)) \in \Lambda(0, R)$ exists iff $R(z)<R \leq 1$, where $R(z)=|z|$. As $R$ increases from $R(z)$ to 1 , the point $v(R)$ moves along the segment $J_{z}$ connecting $\bar{z}$ and $1 / z$ from $\bar{z}$ to $1 / z$ G3 .

Assume now that $\Im z \neq 0$. Denote by $\lambda_{z}$ the arc on the circle passing through $t, 1 / t$ and $\bar{z}$ (and consequently passing through $1 / z$ ) which joins $\bar{z}$ and $1 / z$ and does not contain $t$ and $1 / t$. Let $T(z)$ be such that $z \in b \Delta(T(z), \rho(T(z)))$. For the semiquadric from the second family the intersection $(z, w(T)) \in \Lambda(T, \rho(T))$ exists if and only if $0 \leq T<T(z)$. As $T$ increases from 0 to $T(z), w(T)$ moves along $\lambda_{z}$ from $1 / z$ to $\bar{z}$ G3.

Now, let $z=\eta, 0<\eta<t$. Let $v(R)$ be such that $(\eta, v(R)) \in \Lambda(0, R)$ and let $w(T)$ be such that $(\eta, w(T)) \in \Lambda(T, \rho(T))$. It is easy to see that as $R$ increases from $R(\eta)=\eta$ to $1, v(R) \in \mathbb{R}$ increases from $\eta$ to $1 / \eta$. As $T$ increases from 0 to $\eta$, $w(T) \in \mathbb{R}$ increases from $\eta$ to $1 / \eta$. As $T$ increases from 0 to $\eta, w(T)$ increases from $1 / \eta$ to $\infty$. As $T$ increases from $\eta$ to $T(\eta)$, then $w(T) \in \mathbb{R}$ increases from $-\infty$ to $\eta$.

The situation is entirely different if $z=\eta$, where either $-1<\eta<0$ or $t<\eta<1$. In this case we have "folding": when $R$ increases from $R(\eta)$ to $1, v(R)$ moves from $\eta$ to $1 / \eta$ and when $T$ increases from 0 to $T(\eta)$, then $w(T)$ moves from $1 / \eta$ to $\eta$.

The preceding discussion offers a "fiber" description of $L$ and $N$. We clearly have

$$
L=\bigcup_{z \in \bar{\Delta} \backslash\{0\}}\{z\} \times J_{z}
$$

To describe $N$, recall that so far we have only defined $\lambda_{z}$ for $z \in \bar{\Delta}, \Im z \neq 0$. We now define $\lambda_{z}$ for $z=\eta \in \mathbb{R}$. If $0<\eta<t$, then let $\lambda_{\eta}=(-\infty, \eta] \cup[1 / \eta, \infty)$ and when $-1 \leq \eta<0$, then let $\lambda_{\eta}=[1 / \eta, \eta]$ and when $t<\eta \leq 1$, then let $\lambda_{\eta}=[\eta, 1 / \eta]$. 
Finally, let $\lambda_{0}=(-\infty, 0]$, and $\lambda_{t}=\{t\}$. Then it is easy to see that

$$
N=\bigcup_{z \in \bar{\Delta} \backslash\{t\}}\{z\} \times \lambda_{z}
$$

\section{The DOMAins Bounded By $L$ AND $N$}

Both $L$ and $N$ are smooth, unbounded surfaces with boundary. They have a common boundary consisting of $\{(\zeta, \bar{\zeta}): \quad \zeta \in \bar{\Delta} \backslash\{0, t\}\}$ and $\Lambda(0,1)$. They have no other common points of the form $(z, w)$, where $z \in \bar{\Delta} \backslash[(-1,0] \cup[t, 1)]$. The situation is different for the points $z \in(-1,0) \cup(t, 1)$. In this case $J_{z}=\lambda_{z}$. If $\eta \in(-1,0) \cup(t, 1)$, then $J_{\eta}=\lambda_{\eta}$ is the segment joining $\eta$ and $1 / \eta$.

For each $z \in \Delta, \Im z \neq 0, J_{z} \cup \lambda_{z}$ is a simple closed curve bounding a domain that we denote by $D_{z}$. If $\Im z>0$, then $D_{z}$ is contained in the lower halfplane and if $\Im z<0$, then $D_{z}$ is contained in the upper halfplane. When $z \in \Delta$ approaches a point $a \in b \Delta, \Im a \neq 0$, then the domains $D_{z}$ shrink to the point $\{a\}$. When $z, \Im z>$ 0 , approaches a point $\eta, 0<\eta<t$, then the $D_{z}$ become larger and larger and in the limit they become the lower halfplane. When $z, \Im z<0$, approaches a point $\eta, 0<\eta<t$, then the $D_{z}$ become larger and larger and in the limit they become the upper halfplane. When $z, \Im z>0$, approaches a point $\eta \in(-1,0) \cup(t, 1)$, then the domains $D_{z}$ become thinner and thinner and in the limit they shrink to the segment with endpoints $\eta$ and $1 / \eta$. The same takes place for $\Im z<0$.

As usual, denote by $\pi$ the projection $\pi(z, w)=z$.

We now define two domains in $\mathbb{C}^{2}$ : Let

$$
\Omega_{+}=\bigcup_{z \in \Delta, \Im z>0}\{z\} \times D_{z} \quad \text { and } \quad \Omega_{-}=\bigcup_{z \in \Delta, \Im z<0}\{z\} \times D_{z} .
$$

Clearly $\Omega_{+} \subset\{(z, w): \Im w<0\}$ and $\Omega_{-} \subset\{(z, w): \Im w>0\}$. Obviously

$$
\begin{aligned}
& b \Omega_{+} \cap\{(z, w): \Im z>0\}=(N \cup L) \cap\{(z, w): \Im z>0\} \\
& b \Omega_{-} \cap\{(z, w): \Im z<0\}=(N \cup L) \cap\{(z, w): \Im z<0\} .
\end{aligned}
$$

Further,

$$
b \Omega_{+} \cap \pi^{-1}((0, t))=\bigcup_{0<z<t}[\{z\} \times\{w \in \mathbb{C}: \Im w \leq 0\}]
$$

and

$$
b \Omega_{-} \cap \pi^{-1}((0, t))=\bigcup_{0<z<t}[\{z\} \times\{w \in \mathbb{C}: \Im w \geq 0\}] .
$$

Obviously,

$$
b \Omega_{+} \cap \pi^{-1}((-1,0))=b \Omega_{-} \cap \pi^{-1}((-1,0))=\bigcup_{-1<\eta<0}\{\eta\} \times[1 / \eta, \eta]
$$

and

$$
b \Omega_{+} \cap \pi^{-1}((t, 1))=b \Omega_{-} \cap \pi^{-1}((t, 1))=\bigcup_{t<\eta<1}\{\eta\} \times[\eta, 1 / \eta] .
$$




\section{OUR CR FUnCtion ON $L \cup N$}

Let $n \in \mathbb{N} \cup\{0\}, 0<t<1$ and suppose that $f$ is a continuous function on $\bar{\Delta}$ that satisfies the assumptions of Theorem 4.1.

Define

$$
F(\zeta, \bar{\zeta})=f(\zeta) \quad(\zeta \in \bar{\Delta})
$$

$F$ is a continuous function on $\{(\zeta, \bar{\zeta}): \zeta \in \bar{\Delta}\}$. The assumptions together with the continuity of $F$ and Lemma 4.1 imply that the function $F$ extends from $L \cap \Sigma=$ $\{(\zeta, \bar{\zeta}): \zeta \in \bar{\Delta} \backslash\{0\}\}$ continuously to $L$ such that the extension $\Phi$ is holomorphic on each fiber $\Lambda(0, R)$ of $L$ with a pole of degree $\leq n$ at infinity. Thus, $\Phi$ is a CR function. Similarly, the function $F$ extends from $N \cap \Sigma=\{(\zeta, \bar{\zeta}): \zeta \in \bar{\Delta} \backslash\{t\}\}$ continuously to $N$ so that the extension $\Psi$ is holomorphic on each fiber $\Lambda(T, \rho(T)) \backslash$ $\{(t, 1 / t)\}, 0 \leq T<t$, with a pole of degree $\leq n$ at infinity. Thus, $\Psi$ is a CR function. The functions $\Phi$ and $\Psi$ coincide on $\{(\zeta, \bar{\zeta}): \zeta \in \bar{\Delta} \backslash\{0, t\}\} \cup \Lambda(0,1)$, the common boundary of $L$ and $N$. Since in $\pi^{-1}(\Delta \backslash[(-1,0] \cup[t, 1)])$ there are no other common points of $L$ and $N$ we can define the function $F$ on $M$ where

$$
M=(L \cup N) \backslash[[(-1,0] \cup[t, 1)] \times \mathbb{C}],
$$

which extends the original $F$ and is equal to $\Phi$ on $L$ and to $\Psi$ on $N$. The function $F$ so obtained is continuous on $M$ and holomorphic on each fiber of $M$, so it is a CR function on $M$.

Our final goal will be to show that on $M$ we have

$$
F(z, w)=a_{0}(z)+a_{1}(z) w+\cdots+a_{n}(z) w^{n},
$$

where the $a_{j}, 0 \leq j \leq n$, are holomorphic functions on $\Delta$, which will then imply that

$$
f(z)=F(z, \bar{z})=a_{0}(z)+a_{1}(z) \bar{z}+\cdots+a_{n}(z) \bar{z}^{n}
$$

on $\Delta$, which is the statement of Theorem 4.1.

In exactly the same way as in G2, G3, following an idea of A.Tumanov T1, we now use an argument of $\mathrm{H}$. Lewy $[\mathrm{L}$, extended by $\mathrm{H}$. Rossi $[\mathrm{R}]$, to show that the function $F$ that is $\mathrm{CR}$ on $M$ extends holomorphically through $\{z\} \times D_{z}$ for each $z \in \Delta, \Im z \neq 0$ and that the extension so obtained is holomorphic in $z$. In this way we obtain a function $F$ that is holomorphic on $\Omega_{+}$and on $\Omega_{-}$and which extends continuously to $\bigcup_{z \in \bar{\Delta}, \Im z>0}\{z\} \times b D_{z}$, a part of the boundary of $\Omega_{+}$, and to $\bigcup_{z \in \bar{\Delta}, \Im z<0}\{z\} \times b D_{z}$, a part of the boundary of $\Omega_{-}$.

Before we proceed, notice that our function $F$ is well defined on $(0, t) \times \mathbb{R}$.

We will now show that the continuity of $F$ on $M$ implies that

$$
F \text { extends continuously to } \Omega_{+} \cup[(0, t) \times\{w: \Im w \leq 0\}]
$$

and

$$
F \text { extends continuously to } \Omega_{-} \cup[(0, t) \times\{w: \Im w \geq 0\}] .
$$

We will also show that our assumptions imply that for each $\eta, 0<\eta<t$ there is a constant $c(\eta)$ such that

$$
|F(\eta, \zeta)| \leq c(\eta)(1+|\zeta|)^{n} \quad(\zeta \in \mathbb{C}) .
$$

Suppose for a moment that we have done this.

Since $F$ is holomorphic on $\Omega_{+} \cup \Omega_{-}$it follows that the extended function $F$ is holomorphic on $[\{\eta\} \times\{\Im \zeta>0\}] \cup[\{\eta\} \times\{\Im \zeta<0\}]$ and continuous on $\{\eta\} \times \mathbb{C}$ for 
each $\eta, 0<\eta<t$, so for each such $\eta$, the function $F$ is holomorphic on $\{\eta\} \times \mathbb{C}$. By (8.3) it follows that for each $\eta, 0<\eta<t, w \mapsto F(\eta, w)$ is a polynomial of degree $\leq n$.

\section{Polynomial in the Second variable}

The fact that for each $\eta, 0<\eta<t$, the function $w \mapsto F(\eta, w)$ is a polynomial of degree $\leq n$ implies, for instance, that for each such $\eta$,

$$
\frac{\partial^{k} F}{\partial w^{k}}(\eta, w) \equiv 0 \quad(k \geq n+1, \Im w<0) .
$$

For each $\eta, 0<\eta<t$, and for each $w_{0}, \Im w_{0}<0$, there is a neighbourhood $U \subset \mathbb{C}$ of $\eta$ and a neighbourhood $W$ of $w_{0}$, such that for each $k, \frac{\partial^{k} F}{\partial w^{k}}$ is continuous on $\{z \in U, \Im z \geq 0\} \times W$ (a consequence of expressing the derivatives with the Cauchy integral formula) and holomorphic on $\{z \in U: \Im z>0\} \times W$. By the preceding discussion, for each $k \geq n+1, \frac{\partial^{k} F}{\partial w^{k}}$ vanishes identically on $\{z \in U, \Im z=0\} \times W$, so it follows that it vanishes identically on $\{z \in U, \Im z>0\} \times W$, an open subset of $\Omega_{+}$, and since it is holomorphic on $\Omega_{+}$, it follows that it vanishes identically on $\Omega_{+}$as $\Omega_{+}$is connected. Thus,

$$
\frac{\partial^{k} F}{\partial w^{k}}(z, w)=0 \quad(k \geq n+1) \quad \text { for all }(z, w) \in \Omega_{+} .
$$

In the same way we prove that (9.1) holds for all $z \in \Omega_{-}$. Recall that for each $z \in \Delta, \Im z \neq 0, D_{z}$ is connected and hence (9.1) implies that for each such $z$, there are numbers $a_{0}(z), \ldots, a_{n}(z)$ such that

$$
F(z, w)=a_{0}(z)+a_{1}(z) w+\cdots+a_{n}(z) w^{n} \quad\left(w \in D_{z}\right) .
$$

Since $F$ is holomorphic on $\Omega_{+}$it follows that $z \mapsto a_{j}(z)(0 \leq j \leq n)$ are holomorphic on $\{z \in \Delta, \Im z>0\}$. In the same way we see that $a_{0}, \ldots, a_{n}$ are holomorphic on $\{z \in \Delta: \Im z<0\}$.

Choose distinct points $w_{0}, w_{1}, \ldots, w_{n} \in\{\Im w<0\}$. We have assumed (8.1); hence $F$ extends continuously to $\Omega_{+} \cup[(0, t) \times\{\Im w \leq 0\}]$ and it follows that there is a neighbourhood $U$ of $(0, t)$ in $\mathbb{C}$ such that for each $j, 0 \leq j \leq n$, the function $z \mapsto$ $F\left(z, w_{j}\right)$ extends continuously from $\{z \in U, \Im z>0\}$ to $\{z \in U, \Im z>0\} \cup(0, t)$. Since

$$
\left[\begin{array}{c}
a_{0}(z) \\
\cdots \\
a_{n}(z)
\end{array}\right]=\left[\begin{array}{cccc}
1 & w_{0} & \cdots & w_{0}^{n} \\
& & \cdots & \\
1 & w_{n} & \cdots & w_{n}^{n}
\end{array}\right]^{-1} \cdot\left[\begin{array}{c}
F\left(z, w_{0}\right) \\
\cdots \\
F\left(z, w_{n}\right)
\end{array}\right],
$$

where the inverse of the Vandermonde matrix on the right exists since $w_{j}, 0 \leq j \leq n$ are distinct, it follows that each $a_{j}$ extends continuously from $\{z \in \Delta: \Im z>0\}$ to $\{z \in \Delta: \Im z>0\} \cup(0, t)$. In the same way we see that the functions $a_{0}, \ldots, a_{n}$ extend continuously from $\{z \in \Delta, \Im z<0\}$ to $\{z \in \Delta, \Im z<0\} \cup(0, t)$.

Recall that for each $\eta \in(0, t)$ and $s \in \mathbb{R}, F(\eta, s)$ is well defined and for all $s \in \mathbb{R}$ we have

$$
F(\eta, s)=a_{0}^{+}(\eta)+a_{1}^{+}(\eta) s+\cdots+a_{n}^{+}(\eta) s^{n} \quad(s \in \mathbb{R}),
$$

where the $a_{j}^{+}$are continuous extensions of $a_{j}$ from $\{\zeta \in \Delta$, $\zeta>0\}$ and

$$
F(\eta, s)=a_{0}^{-}(\eta)+a_{1}^{-}(\eta) s+\cdots+a_{n}^{-}(\eta) s^{n} \quad(s \in \mathbb{R}),
$$


where the $a_{j}^{-}$are continuous extensions of $a_{j}$ from $\{\zeta \in \Delta$, $\zeta \zeta<0\}$. Now, (9.4) and (9.3) imply that $a_{j}^{-} \equiv a_{j}^{-}$on $(0, t)$ for each $j, 0 \leq j \leq n$, which implies that there are holomorphic functions $a_{j}, 0 \leq j \leq n$, on $\Delta \backslash[(-1,0] \cup[t, 1)]$, such that $F(z, w)=a_{0}(z)+a_{1}(z) w+\cdots+a_{n}(z) w^{n}$ for all $(z, w) \in \Omega_{-} \cup \Omega_{+} \cup[(0, t) \times \mathbb{C}]$.

In the next section we show first that the functions $a_{j}, 0 \leq j \leq n$, extend holomorphically also across the intervals $(-1,0)$ and $(t, 1)$ and then we show that the remaining points 0 and $t$ are removable singularities for all these functions.

\section{Analyticity of the COEFFiCients}

Our extended function $F$ is well defined on both

$$
\bigcup_{z \in \Delta_{+}}\{z\} \times \bar{D}_{z} \text { and } \bigcup_{z \in \Delta_{-}}\{z\} \times \bar{D}_{z}
$$

where $\Delta_{+}=\{\zeta \in \Delta: \Im z>0\}$ is the upper half of $\Delta$ and $\Delta_{-}$is the lower half of $\Delta$.

For each $z \in \Delta, \Im z \neq 0$, denote by $I_{z}$ the segment from $\bar{z}$ to $1 / z$ (the straight part of $\left.b D_{z}\right)$ and by $J_{z}$ the circular arc part of $b D_{z}$. The sets $I_{z}$ and $J_{z}$ meet at the points $\bar{z}$ and $1 / z$. For $-1<x<0$ we define $I_{x}=J_{x}=(1 / x, x)$. Since the points of $\{x\} \times I_{x}$ belong to both families of semiquadrics there is a problem of defining $F$ on $\{x\} \times I_{x}$. At each point of $\{x\} \times I_{x}$ we have two different values of $F$ : one comes from values of extensions on the first family and the other from the values of extensions on the second family. From the definition of $F$ we know that

$$
\text { the function } F \text { extends continuously to } \bigcup_{z \in \Delta_{+} \cup \Delta_{-} \cup(-1,0)}\{z\} \times I_{z} \text {. }
$$

For $z=x \in(-1,0)$ denote this extension by $\varphi(x, w) \quad(w \in(1 / x, x))$. Clearly $\varphi(x, w)$ is the value of the extension of the original $F$ to the semiquadric $\Lambda(0, R)$ of the first family, which passes through $(x, w)$, at the point $(x, w)$. Similarly,

$$
\text { the function } F \text { extends continuously to } \bigcup_{z \in \Delta_{+} \cup \Delta_{-} \cup(-1,0)}\{z\} \times J_{z} \text {. }
$$

For $z=x \in(-1,0)$ denote the extension by $\psi(x, w)(w \in(1 / x, x))$. Here $\psi(x, w)$ is the value of the extension of the original $F$ to the semiquadric $\Lambda(T, \rho(T))$ of the second family which passes through $(x, w)$, at the point $(x, w)$.

We will show that $\varphi$ and $\psi$ coincide and that for each $x \in(-1,0)$ there are numbers $p_{0}(x), \ldots, p_{n}(x)$, such that

$$
\varphi(x, y)=\psi(x, y)=p_{0}(x)+p_{1}(x) y+\cdots+p_{n}(x) y^{n} \quad(1 / x<y<x),
$$

and we will then show that for each $x \in(0,1)$ and for each $j, 0 \leq j \leq n, p_{j}(x)$ is the value at $x$ of the continuous extension of $a_{j}$ from $\Delta_{+} \cup \Delta_{-}$to $\Delta_{+} \cup \Delta_{-} \cup\{x\}$.

We need the following

Lemma 10.1. Let $p_{m}(w)=a_{m 0}+a_{m 1} w+\cdots+a_{m n} w^{n}$ be a sequence of polynomials. Let $w_{m i}, 1 \leq i \leq n+1$, be sequences of points in $\mathbb{C}$, converging to distinct points $w_{1}, \ldots, w_{n+1}$, respectively. Suppose that for each $i, 1 \leq i \leq n+1$, the sequence $p_{m}\left(w_{m i}\right)$ converges. Then there are $\alpha_{0}, \ldots, \alpha_{n}$ such that $a_{m i}$ converges to $\alpha_{i}$ for each $i, 0 \leq i \leq n$, and therefore, the sequence $p_{m}$ converges, uniformly on compacta, to the polynomial $p(w)=\alpha_{0}+\alpha_{1} w+\cdots+\alpha_{n} w^{n}$. In particular, the $p_{m}\left(w_{m i}\right)$ converge to $p\left(w_{i}\right), 1 \leq i \leq n+1$, for any sequences $w_{m i}$ converging to $w_{i}, 1 \leq i \leq n+1$. 
Proof. Since $w_{1}, \ldots, w_{n+1}$ are distinct, the Vandermonde matrix

$$
V\left(w_{1}, \ldots, w_{m+1}\right)=\left[\begin{array}{cccc}
1 & w_{1} & \cdots & w_{1}^{n} \\
& \cdots & \\
1 & w_{n+1} & \cdots & w_{n+1}^{n}
\end{array}\right]
$$

is nonsingular and consequently for all sufficiently large $m$ the matrices

$$
V\left(w_{m 1}, \ldots, w_{m, n+1}\right)=\left[\begin{array}{ccc}
1 w_{m 1} & \cdots & w_{m 1}^{n} \\
\cdots & \\
1 w_{m, n+1} & \cdots & w_{m, n+1}^{n}
\end{array}\right]
$$

are nonsingular and, as $m \rightarrow \infty$, converge to the nonsingular matrix $V\left(w_{1}, \ldots, w_{n}\right)$. Consequently, for $m$ sufficiently large, the matrices $V\left(w_{m 1}, \ldots, w_{m, n+1}\right)^{-1}$ are well defined and, as $m \rightarrow \infty$, they converge to $V\left(w_{1}, \ldots, w_{n+1}\right)^{-1}$. Since

$$
\left[\begin{array}{c}
p_{m}\left(w_{m 1}\right) \\
\ldots \\
p_{m}\left(w_{m, n+1}\right)
\end{array}\right]=V\left(w_{m 1}, \ldots, w_{m n}\right) \cdot\left[\begin{array}{c}
a_{m 0} \\
\ldots \\
a_{m n}
\end{array}\right]
$$

it follows that

$$
\left[\begin{array}{c}
a_{m 0} \\
\cdots \\
a_{m n}
\end{array}\right]=V\left(w_{m 1}, \ldots, w_{m n}\right)^{-1}\left[\begin{array}{c}
p_{m}\left(w_{m, n+1}\right) \\
\ldots \\
p_{m}\left(w_{m, n+1}\right)
\end{array}\right]
$$

and since both factors on the right converge it follows that the columns on the left converge. This completes the proof.

Now, fix $x,-1<x<0$, and fix distinct points $y_{1}, \ldots, y_{n+1} \in(1 / x, x)$. Choose a sequence $z_{n}, \Im z_{n}>0$, converging to $x$ and observe that by the nature of $b D_{z_{n}}$ for each $i, 1 \leq i \leq n+1$, there is a sequence $u_{m i} \in I_{z_{m}}, m \in \mathbb{N}$ that converges to $y_{i}$, and a sequence $w_{m i} \in J_{z_{m}}$ that converges to $y_{i}$. By (10.1), $F\left(z_{m}, u_{m i}\right)$ converges to $\varphi\left(x, y_{i}\right)$ and by $(10.2), F\left(z_{m}, w_{m i}\right)$ converges to $\psi\left(x, y_{i}\right)$. Now apply Lemma 10.1 to the sequence of polynomials

$$
w \mapsto p_{m}(w)=F\left(z_{m}, w\right)=a_{0}\left(z_{m}\right)+a_{1}\left(z_{m}\right) w+\cdots+a_{n}\left(z_{m}\right) w^{n}
$$

to see that there are numbers $\alpha_{i}=\lim _{m \rightarrow \infty} a_{i}\left(z_{m}\right), 0 \leq i \leq n$ and that for each $i, 1 \leq i \leq n+1$,

$$
\varphi\left(x, y_{i}\right)=\alpha_{0}+\alpha_{1} y_{i}+\cdots+\alpha_{n} y_{i}^{n}=\psi\left(x, y_{i}\right) .
$$

In particular, since the $y_{j}$ were arbitrary, it follows that

$$
\varphi(x, y) \equiv \psi(x, y) \quad(1 / x<y<x) .
$$

Moreover, keeping $y_{1}, \ldots, y_{n+1}$ fixed we see that $\alpha_{0}, \alpha_{1}, \ldots, \alpha_{n}$ do not depend on $z_{n}$ converging to $x$. This implies that each function $z \mapsto a_{i}(z)$ extends continuously to $\Delta_{+} \cup\{x\}$ and thus

$$
\lim _{z \rightarrow x, \Im z>0} F(z, y)=\alpha_{0}+\alpha_{1} y+\cdots+\alpha_{n} y^{n}=\varphi(x, y)=\psi(x, y) .
$$

In the same way we get

$$
\lim _{z \rightarrow x, \Im z<0} F(z, y)=\beta_{0}+\beta_{1} y+\cdots+\beta_{n} y^{n}=\varphi(x, y)=\psi(x, y),
$$


which, finally, implies that each $a_{j}, 0 \leq j \leq n$, extends holomorphically across $(-1,0)$. In the same way we get that each $a_{j}, 0 \leq j \leq n$, extends holomorphically across $(t, 1), F$ is well defined and

$$
F(z, w)=a_{0}(z)+a_{1}(z) w+\cdots+a_{n}(z) w^{n} \quad(z \in \Delta \backslash\{0, t\}) .
$$

\section{Removable singularities of the Coefficients at 0 And at $t$}

In the preceding section we proved that

$$
f(z)=F(z, \bar{z})=a_{0}(z)+a_{1}(z) \bar{z}+\cdots+a_{n}(z) \bar{z}^{n} \quad(z \in \Delta \backslash\{0, t\}),
$$

where the functions $a_{j}, 0 \leq j \leq n$, are holomorphic on $\Delta \backslash\{0, t\}$. Recall that our $f$ has the property that

$$
\left.\begin{array}{l}
\text { for each } \Gamma \in \mathcal{C}_{0} \cup \mathcal{C}_{t} \text { the function } z \mapsto(z-c(\Gamma))^{n} f(z) \\
\text { extends holomorphically from } \Gamma \text {. }
\end{array}\right\}
$$

Proposition 11.1. Suppose that $f$ is as above and suppose that (11.1) holds. Then the functions $a_{j}, 0 \leq j \leq n$, extend holomorphically through $\Delta$.

Proof. If $\Gamma=b \Delta(0, R)$, then $\bar{z}=R^{2} / z$, so the function

$$
z^{n}\left[a_{0}(z)+\frac{a_{1}(z) R^{2}}{z}+\cdots+\frac{a_{n}(z) R^{2 n}}{z^{n}}\right]
$$

provides the holomorphic extension of $z^{n} f(z)$ from $\Gamma$ to $\Delta(0, R) \backslash\{0\}$ if $0<R<t$ and to $\Delta(0, R) \backslash\{0, t\}$ if $t<R<1$.

Consider first the second case. In particular, when $t<R<1$ the function (11.2) has no singularity at $t$, which is the same as to say that the function

$$
z \mapsto a_{0}(z)+\frac{a_{1}(z)}{z} R^{2}+\cdots+\frac{a_{n}(z)}{z^{n}} R^{2 n}
$$

has no singularity at $t$. Let $p_{j}(z)$ be the singular part of $\frac{a_{j}(z)}{z^{j}}$ in the Laurent expansion around $t$. We must have

$$
p_{0}(z)+R^{2} p_{1}(z)+\cdots+R^{2 n} p_{n}(z) \equiv 0 \quad(t<R<1)
$$

for $z$ in a neighbourhood of $t$, which implies that $p_{j}(z) \equiv 0(0 \leq j \leq n)$, so $z \mapsto a_{j}(z)$ is holomorphic in a neighbourhood of $t$, which, since $t>0$, implies that each $a_{j}, 0 \leq j \leq n$, is holomorphic in a neighbourhood of $t$. This shows that each $a_{j}, 0 \leq j \leq n$, is holomorphic on $\Delta \backslash\{0\}$.

Now observe that for each $R, 0<R<1$, the function

$$
\begin{aligned}
z & \mapsto z^{n}\left[a_{0}(z)+a_{1}(z) \frac{R^{2}}{z}+\cdots+a_{n}(z) \frac{R^{2 n}}{z^{n}}\right] \\
& =a_{0}(z) z^{n}+a_{1}(z) R^{2} z^{n-1}+\cdots+a_{n}(z) R^{2 n}
\end{aligned}
$$

provides the holomorphic extension of $z^{n} f$ from $b \Delta(0, R)$ to $\Delta(0, R) \backslash\{0\}$. By our assumption this extension must be holomorphic at 0 . In the Laurent series at the origin, let

$q_{0}$ be the singular part of $z^{n} a_{0}$,

$q_{1}$ be the singular part of $z^{n-1} a_{1}$,

...

$q_{n}$ be the singular part of $a_{n}$. 
We must have

$$
q_{0}+R^{2} q_{1}+\cdots+R^{2 n} q_{n} \equiv 0 \quad(0<R<1),
$$

which implies that

$$
q_{0} \equiv q_{1} \equiv \cdots \equiv q_{n} \equiv 0,
$$

and it follows that there are holomorphic functions $h_{0}, h_{1}, \ldots, h_{n}$ on $\Delta$ such that on $\Delta \backslash\{0\}$ our function $f$ has the form

$$
f(z)=\frac{h_{0}(z)}{z^{n}}+\frac{h_{1}(z)}{z^{n-1}} \bar{z}+\cdots+h_{n}(z) \bar{z}^{n} .
$$

We must now show that each $a_{j}$ has a removable singularity at the origin. To do this, we use the fact that from each circle $b \Delta(\lambda, \rho(\lambda)) \in C_{t}$ the function $f$ extends holomorphically through $\Delta(\lambda, \rho(\lambda)) \backslash\{\lambda\}$ with a pole at $\lambda$. On $b \Delta(\lambda, \rho(\lambda))$ we have

$$
\bar{z}=\lambda+\frac{\rho(\lambda)^{2}}{z-\lambda} \quad(0 \leq \lambda<t)
$$

so our requirement will be that

$$
\frac{h_{0}(z)}{z^{n}}+\frac{h_{1}(z)}{z^{n-1}}\left[\lambda+\frac{\rho(\lambda)^{2}}{z-\lambda}\right]+\cdots+h_{n}(z)\left[\lambda+\frac{\rho(\lambda)^{2}}{z-\lambda}\right]^{n}
$$

must have no pole at the origin. This is the same as to say that

$$
h_{0}(z)+h_{1}(z)\left[z\left(\lambda+\frac{\rho\left(\lambda^{2}\right.}{z-\lambda}\right)\right]+\cdots+h_{n}(z)\left[z\left(\lambda+\frac{\rho\left(\lambda^{2}\right.}{z-\lambda}\right)\right]^{n}
$$

has a zero of order $\geq n$ at 0 for each $\lambda, 0 \leq \lambda<t$. Requiring this we should then conclude that

$$
\begin{aligned}
& h_{0} \text { has a zero of order } \geq n \text { at the origin, } \\
& h_{1} \text { has a zero of order } \geq n-1 \text { at the origin, } \\
& \ldots \\
& h_{n-1} \text { has a zero of order } \geq 1 \text { at the origin, }
\end{aligned}
$$

which is the same as to conclude that each of the functions $H_{0}=h_{0}, H_{1}=$ $z h_{1}, \ldots, H_{n}=z^{n} h_{n}$ has a zero of order $\geq n$ at the origin. Thus, denoting

$$
\varphi(z, \lambda)=\lambda+\frac{\rho(\lambda)^{2}}{(z-\lambda)}
$$

we have to show that if

$$
H_{0}(z)+H_{1}(z) \varphi(z, \lambda)+\cdots+H_{n}(z) \varphi(z, \lambda)^{n}
$$

has, for each $\lambda$, a zero of order $\geq n$ at the origin, then the same holds for each $H_{j}$. Suppose that (11.3) has a zero at the origin for each $\lambda$. This implies that

$$
H_{0}(0)+H_{1}(0) \varphi(0, \lambda)+H_{2}(0) \varphi(0, \lambda)^{2}+\cdots+H_{n}(0) \varphi(0, \lambda)^{n} \equiv 0 .
$$

We now use the fact that

$$
\left\{\lambda-\frac{\rho(\lambda)^{2}}{\lambda}: 0<\lambda<t\right\}
$$

is a large set. This is so by the continuity: when $\lambda \rightarrow 0, \lambda-\rho(\lambda)^{2} / \lambda$ is very large and when $\lambda$ is near $t, \rho(\lambda)$ is near 0 , so $\lambda-\rho(\lambda)^{2} / \lambda$ is near $t$. Thus, (11.4) implies 
that $H_{0}(0)=\cdots=H_{n}(0)=0$, so $H_{0}(z)=z G_{0}(z), \ldots, H_{n}(z)=z G_{n}(z)$ with $G_{i}$ holomorphic. If (11.3), which now becomes

$$
z\left[G_{0}(z)+G_{1}(z) \varphi(z, \lambda)+\cdots+G_{n}(z) \varphi(z, \lambda)^{n}\right],
$$

has a zero of order $\geq n$ at the origin, then

$$
G_{0}(z)+G_{1}(z) \varphi(z, \lambda)+\cdots+G_{n}(z) \varphi(z, \lambda)^{n}
$$

has a zero of order $\geq n-1$ at the origin. In particular,

$$
G_{0}(0)+G_{1}(0) \varphi(0, \lambda)+\cdots+G_{n}(0) \varphi(0, \lambda)^{n} \equiv 0
$$

and so, after $n$ steps we deduce that each $H_{j}$ has zero of order $\geq n$ at the origin. This completes the proof of Theorem 4.1 and thus proves Theorem 1.1 in the case when $\beta=0$ and $\alpha \in \Delta \backslash\{0\}$, assuming that we have proved (8.1), (8.2) and $(8.3)$.

\section{Proving (8.1), (8.2) And (8.3), Part 1}

We first look at (8.1), that is, at the function $F$ on $\Omega_{+}$. Write

$$
M_{+}=\{(z, w) \in M: \Im z \geq 0\} .
$$

Clearly $M_{+}$is a part of $b \Omega_{+}$. We have

$$
M_{+}=\left[\bigcup_{z \in \bar{\Delta}, \Im z>0}\{z\} \times b D_{z}\right] \cup\left[\bigcup_{z \in(0, t)}\{z\} \times \mathbb{R}\right] .
$$

We know that the function $F$ is well defined and continuous on $M_{+}$. We also know that the function $F$ is continuous on

$$
\bigcup_{z \in \bar{\Delta}, \Im z>0} \bar{D}_{z}=\Omega_{+} \cup\left[\bigcup_{z \in \bar{\Delta}, \Im z>0}\{z\} \times b D_{z}\right]
$$

and that the extension is holomorphic on $\Omega_{+}$. We now want to show that $F$ extends continuously to $\Omega_{+} \cup M_{+} \cup \Theta_{+}$, where

$$
\Theta_{+}=\bigcup_{0<\eta<t}\{\eta\} \times\{\Im \zeta \leq 0\} .
$$

The set $\Theta_{+}$is a disjoint union of halfplanes attached to $M_{+}$along $\bigcup_{z \in(0, t)}\{z\} \times \mathbb{R}$ and is contained in $b \Omega_{+}$. In other words, we want to show that our function, holomorphic on $\Omega_{+}$, extends continuously to

$$
\left[\bigcup_{z \in \bar{\Delta}, \Im z>0}\{z\} \times \overline{D_{z}}\right] \cup\left[\bigcup_{0<\eta<t}\{\eta\} \times\{\Im \zeta \leq 0\}\right],
$$

which is (8.1) that we want to prove. This will imply that for each $\eta \in(0, t)$ the function $\zeta \mapsto F(\eta, \zeta)$ is continuous on $\{\Im \zeta \leq 0\}$ and holomorphic on $\{\Im \zeta<0\}$. We will also show that it satisfies an estimate of the form

$$
|F(\eta, \zeta)| \leq M_{\eta}(1+|\zeta|)^{n} \quad(\Im \zeta \leq 0),
$$

which will, after proving the inequality also for $\Im \zeta \geq 0$, give (8.3). To get an estimate of the form (12.1) we first prove the following. 
Lemma 12.1. Given $\eta, 0<\eta<t$ there are an open disc $U$ centered at $\eta$ and a constant $M=M(U)$ such that

$$
\left|\frac{1}{(w-i)^{n}} F(z, w)\right| \leq M \quad\left(z \in U, \Im z>0, w \in b D_{z}\right) .
$$

Remark. Note that for $z \in \Delta, \Im z>0, \overline{D_{z}}$ is contained in $\{\Im w<0\}$, where the function $w \mapsto 1 /(w-i)$ is holomorphic.

Remark. By the maximum principle the estimate (12.2) implies that

$$
\left|\frac{1}{(w-i)^{n}} F(z, w)\right| \leq M \quad\left(z \in U, \Im z>0, w \in \overline{D_{z}}\right),
$$

which gives

$$
|F(z, w)| \leq M(1+|w|)^{n} \quad\left(z \in U, \Im z>0, w \in \overline{D_{z}}\right)
$$

and which, after proving the desired continuous extendibility, gives (12.1).

Proof. Note first that $w \mapsto 1 /(w-i)$ is holomorphic on $\{\Im w \leq 0\}$ and satisfies $|1 /(w-i)| \leq 1(\Im w \leq 0)$. Fix $\eta, 0<\eta<t$. There are an open disc $U$ centered at $\eta$ and $T_{0}$ and $R_{0}$ such that

$$
U \cap \bar{\Delta}(T, \rho(T))=\emptyset \quad\left(T_{0}<T<\eta\right) \text { and } U \cap \bar{\Delta}(0, R)=\emptyset \quad\left(0<R<R_{0}\right) .
$$

In other words, if $z \in U$ and $\{z\} \times \mathbb{C}$ meets $\Lambda(T, \rho(T)) \cup b \Lambda(T, \rho(T))$, then necessarily $0 \leq T \leq T_{0}$, and if $\{z\} \times \mathbb{C}$ meets $\Lambda(0, R) \cup b \Lambda(0, R)$, then necessarily $R_{0} \leq R \leq 1$.

Recall that $\Lambda(T, \rho(T))=\left\{\left(\zeta, T+\rho(T)^{2} /(\zeta-T)\right): 0<|\zeta-T|<\rho(T)\right\}$ and $\Lambda(0, R)=\left\{\left(\zeta, R^{2} / \zeta\right): 0<|\zeta|<R\right\}$ and notice that for $w=T+\rho(T)^{2} /(z-T)$ we have $1 /(w-i)=(z-T) /\left[(T-i)(z-T)+\rho(T)^{2}\right]$ and for $w=R^{2} / z$ we have $1 /(w-i)=z /\left(R^{2}-i z\right)$.

Choose $\varepsilon>0$ so small that $\varepsilon<\rho\left(T_{0}\right)^{2} /(2 \sqrt{2})$ and $\varepsilon<R_{0}^{2} / 2$. Assume that $z \in U, \Im z>0$. If $\{z\} \times \mathbb{C}$ meets $\Lambda(T, \rho(T)) \cup b \Lambda(T, \rho(T))$, then $0 \leq T \leq T_{0}$ and $\rho(T) \geq \rho\left(T_{0}\right)$, so if $|z-T|<\varepsilon$, then

$$
\left|\rho(T)^{2}+(T-i)(z-T)\right| \geq \rho\left(T_{0}\right)^{2}-\sqrt{2} \varepsilon \geq \rho\left(T_{0}\right)^{2} / 2 .
$$

If $\{z\} \times \mathbb{C}$ meets $\Lambda(0, R) \cup b \Lambda(0, R)$ it follows that $1 \geq R \geq R_{0}$ and consequently, for $|z|<\varepsilon$ we have $\left|R^{2}-i z\right| \geq R_{0}^{2}-\varepsilon \geq R_{0}^{2} / 2$.

Recall that on $\Lambda(T, \rho(T)$ we have

$$
F\left(z, T+\frac{\rho(t)^{2}}{z-T}\right)=\frac{d_{-n}(T)}{(z-T)^{n}}+\cdots+\frac{d_{-1}(T)}{z-T}+h_{T}\left(\frac{z-T}{\rho(T)}\right),
$$

where, by Lemma 4.1 , the functions $d_{-1}, \ldots, d_{-n}$ are continuous and $h_{T}$ are functions from the disc algebra, continuously depending on $T$. On $\Lambda(0, R)$ we have

$$
F\left(z, \frac{R^{2}}{z}\right)=\frac{c_{-n}(R)}{z^{n}}+\cdots+\frac{c_{-1}(R)}{z}+g_{R}\left(\frac{z}{R}\right)
$$

where, by Lemma 4.1, the functions $c_{-1}, \ldots, c_{-n}$ are continuous and $g_{R}$ are functions from the disc algebra, continuously depending on $R$.

Fix $z \in U, \Im z>0$, and fix $T$ such that $\{z\} \times \mathbb{C}$ meets $\Lambda(T, \rho(T) \cup b \Lambda(T, \rho(T))$. Let $(z, w(z)) \in \Lambda\left(T, \rho(T) \cup b \Lambda(T, \rho(T))\right.$. We know that this implies that $0 \leq T \leq T_{0}$, 
so $1 \geq \rho(T) \geq \rho\left(T_{0}\right)$. Suppose first that $|z-T|<\varepsilon$. Then

$$
\begin{aligned}
& \left|\left(\frac{1}{w(z)-i}\right)^{n} F(z, w(z))\right| \\
& =\left|\left[\frac{z-T}{(T-i)(z-T)+\rho(T)^{2}}\right]^{n} \cdot\left[\frac{d_{-n}(T)}{(z-T)^{n}}+\cdots+\frac{d_{-1}(T)}{z-T}+h_{T}\left(\frac{z-T}{\rho(T)}\right)\right]\right| \\
& =\left|\frac{1}{(T-i)(z-T)+\rho(T)^{2}}\right|^{n} \\
& \cdot\left|d_{-n}(T)+d_{-n+1}(T)(z-T)+\cdots+d_{-1}(T)(z-T)^{n-1}+(z-T)^{n} \cdot h_{T}\left(\frac{z-T}{\rho(T)}\right)\right| \\
& \leq\left[\frac{2}{\rho\left(T_{0}\right)^{2}}\right]^{n} \cdot\left[\left|d_{n}(T)\right|+\cdots+\left|d_{-1}(T)\right| \varepsilon^{n-1}+\varepsilon^{n}\left|h_{T}\left(\frac{z-T}{\rho(T)}\right)\right|\right] .
\end{aligned}
$$

Since $|1 /(w(z)-i)| \leq 1$ it follows that if $|z-T| \geq \varepsilon$, then

$$
\left|\left(\frac{1}{w(z)-i}\right)^{n} F(z, w(z))\right| \leq \frac{\left|d_{-n}(T)\right|}{\varepsilon^{n}}+\cdots+\frac{\left|d_{-1}(T)\right|}{\varepsilon}+\left|h_{T}\left(\frac{z-T}{\rho(T)}\right)\right| .
$$

Since the functions $d_{j},-n \leq j \leq-1$ are continuous and since $h_{T}$, the functions in the disc algebra, continuously depend on $T$ the right hand sides of both inequalities above are uniformly bounded for $0 \leq T \leq T_{0}$. It follows that the function $(z, w) \mapsto$ $F(z, w) /(w-i)^{n}$ is bounded on $\bigcup_{z \in U, \Im z>0}\{z\} \times \lambda_{z}$.

Now, let $z \in U, \Im z>0$ and fix $R$ such that $\{z\} \times \mathbb{C}$ meets $\Lambda(0, R) \cup b \Lambda(0, R)$. We know that this implies that $R \geq R_{0}$. Let $(z, w(z)) \in \Lambda(0, R) \cup b \Lambda(0, R)$. As above, suppose first that $|z|<\varepsilon$. Then

$$
\begin{aligned}
& \left|\left(\frac{1}{w(z)-i}\right)^{n} F(z, w(z))\right| \\
& =\left|\left(\frac{z}{R^{2}-i z}\right)^{n}\left[\frac{c_{n}(R)}{z^{n}}+\cdots+\frac{c_{-1}(R)}{z}+g_{R}\left(\frac{z}{R}\right)\right]\right| \\
& =\left|\frac{1}{\left(R^{2}-i z\right)^{n}}\left[c_{-n}(R)+\cdots+c_{-1}(R) z^{n-1}+z^{n} g_{R}\left(\frac{z}{R}\right)\right]\right| \\
& \leq\left(\frac{2}{R_{0}^{2}}\right)^{n} \cdot\left[\left|c_{-n}(R)\right|+\cdots+\left|c_{-1}(R)\right|+\left|g_{R}\left(\frac{z}{R}\right)\right|\right] .
\end{aligned}
$$

Since $|1 /(w(z)-i)| \leq 1$ it follows that if $|z-T| \geq \varepsilon$, then, as above,

$$
\left|\left(\frac{1}{w(z)-i}\right)^{n} F(z, w(z))\right| \leq\left[\frac{\left|c_{-n}(R)\right|}{\varepsilon^{n}}+\cdots+\frac{\left|c_{-1}(R)\right|}{\varepsilon}+\left|g_{R}\left(\frac{z}{R}\right)\right|\right] .
$$

Since the functions $c_{j},-n \leq j \leq-1$ are continuous and since $g_{R}$, the functions in the disc algebra, continuously depend on $R$ the right hand sides of both inequalities above are uniformly bounded for $R_{0} \leq R \leq 1$. It follows that the function $(z, w) \mapsto$ $F(z, w) /(w-i)^{n}$ is bounded on $\bigcup_{z \in U, \Im z>0}\{z\} \times I_{z}$, which completes the proof of Lemma 12.1.

\section{Completing the Proof of (8.1), (8.2) And (8.3)}

We use the map $w \mapsto \varphi(w)=1 /(w-i)$ which maps $\{\Im \zeta \leq 0\}$ homeomorphically to $\overline{\mathbb{P}} \backslash\{i\}$, where $\mathbb{P}$ is the disc in $\mathbb{C}$ centered at $i / 2$ of radius $1 / 2$, and which maps $\{\Im w<0\}$ biholomorphically to $\mathbb{P}$. Note that $\varphi^{-1}(W)=1 / W+i$. Thus, the map 
$(z, w) \mapsto \Phi(z, w)=(z, \varphi(w))$ maps $\mathbb{C} \times\{\Im w \leq 0\}$ homeomorphically to $\mathbb{C} \times(\overline{\mathbb{P}} \backslash\{0\})$ and $\mathbb{C} \times\{\Im w<0\}$ biholomorphically to $\mathbb{C} \times \mathbb{P}$.

The map $\Phi$ maps the domain $\Omega_{+}$biholomorphically to the domain $\Sigma_{+}=\Phi\left(\Omega_{+}\right)$ $=\bigcup_{z \in \Delta, \Im z>0}\{z\} \times E_{z}$, where for each $z \in \Delta, \Im z>0, E_{z}=\varphi\left(D_{z}\right)$ is a domain in $\mathbb{P}$ whose boundary consists of two circular arcs with endpoints $\varphi(\bar{z})$ and $\varphi(1 / z)$. When $z, \Im z>0$, converges to a point $\eta \in(0, t)$, the endpoints converge to the points $\varphi(1 / \eta)$ and $\varphi(\eta)$ on $b \mathbb{P}$ and the domains $E_{z}$ converge to $\mathbb{P}$. Clearly

$$
\Phi\left(M_{+}\right)=\left[\bigcup_{z \in \bar{\Delta}, \Im z>0}\{z\} \times b E_{z}\right] \cup\left[\bigcup_{z \in(0, t)}\{z\} \times(b \mathbb{P} \backslash\{0\})\right] .
$$

We want to prove that $F$ extends continuously to $[U \cap \mathbb{R}] \times\{\Im w \leq 0\}$. This will be done if we prove that the function $W \mapsto F(z, 1 / W+i)$, which is continuous on

$$
\left[\bigcup_{z \in U, \Im z>0}\{z\} \times b E_{z}\right] \cup\left[\bigcup_{z \in U \cap \mathbb{R}}\{z\} \times[b \mathbb{P} \backslash\{0\}]\right]
$$

and which is continuous on

$$
\bigcup_{z \in U, \Im z>0}\{z\} \times \overline{E_{z}}
$$

and holomorphic on $\bigcup_{z \in U, \Im z>0}\{z\} \times E_{z}$, extends continuously to $\bigcup_{z \in U \cap \mathbb{R}}\{z\} \times$ $[\overline{\mathbb{P}} \backslash\{0\}]$. By what we have shown above the function

$$
G(z, W)=\left\{\begin{array}{l}
W^{n+1} F(z, 1 / W+i) \quad(W \neq 0), \\
0(W=0)
\end{array}\right.
$$

is continuous on

$$
\left[\bigcup_{z \in U, \Im z>0}\{z\} \times b E_{z}\right] \cup\left[\bigcup_{z \in U \cap \mathbb{R}}\{z\} \times b \mathbb{P}\right]
$$

and continuous on $\bigcup_{z \in U, \Im z>0}\{z\} \times \overline{E_{z}}$ and holomorphic on $\bigcup_{z \in U, \Im z>0}\{z\} \times E_{z}$. The continuous extendibility of $F$, which we want to prove, will be proved once we have shown that our function $G$ extends continuously to

$$
\left[\bigcup_{z \in U, \Im z>0}\{z\} \times \overline{E_{z}}\right] \cup\left[\bigcup_{\eta \in U \cap \mathbb{R}}\{\eta\} \times \overline{\mathbb{P}}\right]
$$

With no loss of generality assume that $0 \in E_{z}$ for all $z \in U, \Im z>0$. We understand that $E_{z}=\mathbb{P}$ for $z \in U \cap \mathbb{R}$. The domains $E_{z}$ change continuously with $z$ in the sense that the $b E_{z}$ change continuously with $z$ in Fréchet's sense [Ts, p. 383], which, by a theorem of Courant [Ts, p. 383] implies that if for each $z \in U, \Im z \geq 0, \psi_{z}$ is the conformal map from $\Delta$ to $E_{z}, \psi_{z}(0)=0, \psi_{z}^{\prime}(0)>0$, these maps change continuously with $z$, uniformly on $\bar{\Delta}$. It follows that if we set $\Psi(z, w)=\left(z, \psi_{z}(w)\right)$, then $\Psi$ maps $\{z \in U, \Im z \geq 0\} \times \bar{\Delta}$ homeomorphically onto $\bigcup_{z \in U, \Im z \geq 0}\{z\} \times$ $\overline{E_{z}}$. Recall that the function $G$ is continuous on $\bigcup_{z \in U \Im z \geq 0}\{z\} \times b E_{z}$ and is also continuous on $\bigcup_{z \in U, \Im z>0}\{z\} \times \overline{E_{z}}$ and holomorphic on $\bigcup_{z \in U, \Im z>0}\{z\} \times E_{z}$.

Let $J=G \circ \Psi$. The function $J$ is continuous on $\{z \in U, \Im z>0\} \times \bar{\Delta}$ and holomorphic on each $\{z\} \times \Delta, z \in U, \Im z>0$, and is also continuous on $\{z \in$ $U, \Im z \geq 0\} \times b \Delta$. By the continuity it follows that for each $z \in(0, t)$ the function 
$J$ extends holomorphically through $\{z\} \times \Delta$ and if we define the extension $\tilde{J}$ so that on each $\{z\} \times \Delta$ it is the holomorphic extension of $J$ from $\{z\} \times b \Delta$, then the so-extended function will be, by the continuity of $J$ on $\{z \in U, \Im z \geq 0\} \times b \Delta$ and by the maximum principle, continuous on $\bigcup_{z \in U, \Im z \geq 0}\{z\} \times \bar{\Delta}$. It follows that $\tilde{J} \circ \Psi^{-1}$ provides the necessary extension of $G$ to $\bigcup_{z \in U, \Im z>0}\{z\} \times \overline{E_{z}}$.

This completes the proof of (8.1), and "half" of (8.3). In the same way we prove (8.2) and the "other half" of (8.3). Theorem 4.1 is proved. This proves Theorem 1.1 in the case $(2.2)$.

The proof of Theorem 1.1 in the cases (2.3) and (2.4) is almost the same. The case (2.3) is the limiting case of (2.2) when $t$ tends to 1 . For $z \in \Delta, z \notin \mathbb{R}$, the domain $D_{z}$ is now bounded by $I_{z}$, the segment joining $\bar{z}$ and $1 / z$, and by $\lambda_{z}$, the arc of the circle passing through $\bar{z}, 1 / z$ and 1 , with endpoints $\bar{z}$ and $1 / z$, which does not contain 1 . Folding occurs only on the interval $(-1,0)$ and there is only the singularity at the origin to remove in proving the analyticity of the coefficients $a_{0}, \ldots, a_{n}$ on $\Delta$. The case (2.4) is even simpler. The domain $D_{z}$ is now bounded by $\lambda_{z}$ and $\mu_{z}$, where $\mu_{z}$ is the arc on the circle passing through $\bar{z}, 1 / z$ and -1 , with endpoints $\bar{z}$ and $1 / z$, which does not contain -1 . There is no folding and no singularity to remove. Theorem 1.1 is proved.

\section{REMARKS AND OPEN QUESTIONS}

Remark. In Theorem 1.1 the continuity of $f$ at the boundary is essential as shown by the example

$$
f(z)=\frac{z(z-1 / 2)}{1-|z|^{2}} \quad(z \in \Delta)
$$

of a function which extends holomorphically from every circle $\Gamma \in \mathcal{C}_{0} \cup \mathcal{C}_{1 / 2}$, yet it is not holomorphic on $\Delta$.

Remark. Note that if $f$ is continuous on $\bar{\Delta}$ and of the form (1.1), then $a_{j}, 0 \leq j \leq n$, need not be continuous on $\bar{\Delta}$. To see this, let $g$ be a bounded holomorphic function on $\Delta$ that does not extend continuously to $\bar{\Delta}$. Then $f(z)=\left(1-|z|^{2}\right) g(z)=$ $g(z)-[z g(z)] \bar{z}$ extends continuously through $\bar{\Delta}$, yet the functions $g$ and $z g$ do not.

Example 14.1. The function $g(z, w)=|w|^{2}((z, w) \in b \mathbb{B})$ extends holomorphically into $\mathbb{B}$ along each complex line passing through the origin and along each complex line parallel to one of the coordinate axes that meets $\mathbb{B}$. Let $M$ be the Moebius transform in $\mathbb{C}^{2}$ which maps the origin to the point $a=(1 / 2,1 / 2) \mathrm{Ru}$. $M$ maps the complex lines through the origin to $\mathcal{L}(a)$, the complex lines parallel to the $z$-axis to $\mathcal{L}(b)$ and the complex lines parallel to the $w$-axis to $\mathcal{L}(c)$ where $b, c$ are contained in $\mathbb{C}^{2} \backslash \overline{\mathbb{B}}$ and satisfy $\langle a \mid b\rangle=\langle a \mid c\rangle=1,\langle b \mid c\rangle \neq 1$. The function $f=g \circ M^{-1}$ is real analytic on $b \mathbb{B}$ and extends into $\mathbb{B}$ along each complex line $L \in \mathcal{L}(a) \cup \mathcal{L}(b) \cup \mathcal{L}(c)$ yet it does not extend holomorphically into $B$, and so, by the main result of [G3], $a$ is the only point in $\mathbb{B}$ such that $f$ extends holomorphically into $\mathbb{B}$ along each complex line through this point.

This example shows that in Theorem 1.2 and Corollary 1.3 one cannot drop the assumption that $\langle a \mid b\rangle \neq 1$ if one of the points $a, b$ is in $\mathbb{B}$ and the other is in $\mathbb{C}^{2} \backslash \overline{\mathbb{B}}$. It is easy to see that the points $a, b, c$ do not lie on the same complex line, so the example shows also that there are triples $a, b, c$ of points in $\mathbb{C}^{2}$, not lying on the same complex line, such that $\mathcal{L}(a) \cup \mathcal{L}(b) \cup \mathcal{L}(c)$ is not a test family for holomorphic extendibility for $\mathcal{C}^{\infty}(b \mathbb{B})$. 
Example 14.2. Let

$$
g(z, w)= \begin{cases}\frac{z^{k+2}}{\bar{z}} & (z \neq 0), \\ 0 & (z=0) .\end{cases}
$$

This is a function of class $\mathcal{C}^{k}$ on $b \mathbb{B}$ which extends holomorphically into $\mathbb{B}$ along any complex line which meets $\{0\} \times \Delta$. In particular, it extends holomorphically into $\mathbb{B}$ along every complex line that is parallel to the $z$-axis. Let $M$ be the Moebius map in $\mathbb{C}^{2}$ that maps the origin to the point $(1 / 2,0)$. The function $f=g \circ M^{-1}$ extends holomorphically into $\mathbb{B}$ along any complex line that meets $L \cap \mathbb{B}$, where $L=\{(z, w): z=1 / 2\}$, and along each complex line passing through $(2,0)$ yet it does not extend holomorphically through $\mathbb{B}$. Choosing $a, b \in L \cap \mathbb{B}$ and $c=(2,0)$ we have $\langle a \mid b\rangle=\langle a \mid c\rangle=1$, which shows that in Corollary 1.3 we cannot drop the assumption that one of the numbers $\langle a \mid b\rangle,\langle a \mid c\rangle$ is different from 0 .

If $E$ is a complex line that misses $\overline{\mathbb{B}}$, then given a finite set $\left\{a_{1}, a_{2}, \ldots, a_{n}\right\} \subset E$ there is a real analytic function on $b \mathbb{B}$ which extends holomorphically into $\mathbb{B}$ along every $L \in \mathcal{L}\left(a_{1}\right) \cup \mathcal{L}\left(a_{2}\right) \cup \cdots \cup \mathcal{L}\left(a_{n}\right)$, yet it does not extend holomorphically through $\mathbb{B}[\mathrm{KM}, \mathrm{G3}$. The situation in the case when $E$ is tangent to $b \mathbb{B}$ is unclear. If $E$ meets $\mathbb{B}$ the situation is different for functions of class $\mathcal{C}^{\infty}$ : the lines through two points $a_{1}, a_{2}$ suffice provided that $\left\langle a_{1} \mid a_{2}\right\rangle \neq 1$ in the case that one of the points $a_{1}, a_{2}$ is in $\mathbb{B}$ and the other is in $\mathbb{C}^{2} \backslash \overline{\mathbb{B}}[\mathrm{G} 3]$. The situation is different for functions of class $\mathcal{C}^{k}$ : Given numbers $t_{1}, t_{2}, \ldots, t_{n}, 1<t_{1}<t_{2}<\cdots<t_{n}<\infty$ the function

$$
(z, w) \mapsto\left\{\begin{array}{l}
\frac{w^{k+2}}{\bar{w}}\left(z-\frac{1}{t_{1}}\right) \cdots\left(z-\frac{1}{t_{n}}\right) \quad(w \neq 0) \\
0 \quad(w=0)
\end{array}\right.
$$

is a function of class $\mathcal{C}^{k}$ on $b \mathbb{B}$ which extends holomorphically into $B$ along each complex line which meets $\Delta \times\{0\}$ as well as along each complex line $L \in \mathcal{L}\left(\left(t_{1}, 0\right)\right) \cup$ $\mathcal{L}\left(\left(t_{2}, 0\right)\right) \cup \cdots \cup \mathcal{L}\left(\left(t_{n}, 0\right)\right)$ yet does not extend holomorphically through $\mathbb{B}$.

We conclude with the following open

Question. Suppose that $a, b, c \in \mathbb{C}^{2}$ do not lie on the same complex line and assume that $\Lambda(a, b) \cap \overline{\mathbb{B}}=\Lambda(b, c) \cap \overline{\mathbb{B}}=\Lambda(a, c) \cap \overline{\mathbb{B}}=\emptyset$. Is $\mathcal{L}(a) \cup \mathcal{L}(b) \cup \mathcal{L}(c)$ a test family for holomorphic extendibility for $\mathcal{C}(b \mathbb{B})$ ? If not, is this family a test family for holomorphic extendibility for $\mathcal{C}^{\infty}(b \overline{\mathbb{B}})$ ?

\section{ACKNOWLEDGEMENT}

The author is indebted to M. Agranovsky for providing the example in Section 3. A part of this paper was written in September 2010 during the author's stay at the Institute of Mathematics, University of Bern, Switzerland. He wishes to thank Frank Kutzschebauch for the invitation. This paper was supported in part by the ministry of Higher Education, Science and Technology of Slovenia through the research program Analysis and Geometry, Contract No. P1-02091 (B).

\section{REFERENCES}

[A1] M. L. Agranovsky: Analog of a theorem of Forelli for boundary values of holomorphic functions on the unit ball of $C^{n}$, J. d'Analyse Math. 113 (2011) 293-304.

[A2] M. L. Agranovsky: Characterization of polyanalytic functions by meromorphic extensions into chains of circles, J. d'Analyse Math, 113 (2011) 305-329.

[A3] M. L. Agranovsky: Boundary Forelli theorem for the sphere in $\mathbb{C}^{n}$ and $n+1$ bundles of complex lines, http:/arxiv.org/abs/1003.6125. 
[AG] M. L. Agranovsky and J. Globevnik: Analyticity on circles for rational and realanalytic functions of two real variables, J. d'Analyse Math. 91 (2003) 31-65. MR.2037401 (2004j:30003)

[AV] M. L. Agranovskiǔ and R. E. Val'skiǔ: Maximality of invariant algebras of functions, Sibirsk. Mat. Zh., 12 (1971) No. 1, 3-12. MR0285911(44:3128)

[B1] L. Baracco: Holomorphic extension from the sphere to the ball, http://arxiv.org/ abs/0911.2560

[B2] L. Baracco: Separate holomorphic extension along lines and holomorphic extension of a continuous function from the sphere to the ball: solution of a conjecture by M. Agranovsky http://arxiv.org/abs/1003.4705.

[G1] J. Globevnik: Holomorphic extensions from open families of circles, Trans. Amer. Math. Soc. 355 (2003) 1921-1931. MR1953532 (2003j:30007)

[G2] J. Globevnik: Analyticity of functions analytic on circles, Jour. Math. Anal. Appl. 360 (2009) 363-368. MR2561234 (2010k:30018)

[G3] J. Globevnik: Small families of complex lines for testing holomorphic extendibility, To appear in Amer. J Math. http://arxiv.org/abs/0911.5088.

$[\mathrm{H}] \quad$ K. Hoffman, Banach spaces of analytic functions, Prentice Hall, Englewood Cliffs (N.J.), 1962. MR0133008 (24:A2844)

[KM] A. M. Kytmanov and S. G. Myslivets: On families of complex lines sufficient for holomorphic extension, Mathematical Notes 83 (2008) 500-505 (translated from Mat. Zametki 83 (2008) 545-551. MR 2431620 (2009d:32007)

[L] H. Lewy: On the local character of the solutions of an atypical linear differential equation in three variables and a related theorem for regular functions of two complex variables, Ann. of Math. (2) 64 (1956) 514-522. MR0081952 (18:473b)

[R] H. Rossi: A generalization of a theorem of Hans Lewy, Proc. Amer. Math. Soc. 19 (1968) 436-440. MR0222327 (36:5379)

$[\mathrm{Ru}] \mathrm{W}$. Rudin: Function theory in the unit ball of $C^{n}$, Springer, Berlin-Heidelberg-New York, 1980. MR601594 (82i:32002)

[S] E. L. Stout: The boundary values of holomorphic functions of several complex variables, Duke Math. J. 44 (1977) 105-108. MR0437800 (55:10722)

[T1] A. Tumanov: A Morera type theorem in the strip, Math. Res. Lett. 11 (2004) 23-29. MR2046196 (2004k:30004)

[T2] A. Tumanov: Testing analyticity on circles, Amer. J. Math 129 (2007) 785-790. MR.2325103 (2008d:30005)

[Ts] M. Tsuji: Potential theory in modern function theory, Maruzen, Tokyo, 1959. MR0114894 $(22: 5712)$

Institute of Mathematics, Physics and Mechanics, University of Ljubljana, LjublJANa, Slovenia

E-mail address: josip.globevnik@fmf.uni-lj.si 